\title{
A DESCRIPTIVE VIEW OF UNITARY GROUP REPRESENTATIONS
}

\author{
SIMON THOMAS
}

Dedicated to the memory of Greg Hjorth

\begin{abstract}
In this paper, we will study the relative complexity of the unitary duals of countable groups. In particular, we will explain that if $G$ and $H$ are countable amenable non-type I groups, then the unitary duals of $G$ and $H$ are Borel isomorphic.
\end{abstract}

\section{IntroduCtion}

Let $G$ be a countable group. Then the unitary dual $\widehat{G}$ of $G$ is the set of equivalence classes of irreducible unitary representations of $G$, equipped with its Mackey Borel structure. In more detail, for each $n \in\{1,2, \cdots, \infty\}$, let $\operatorname{Irr}_{n}(G)$ be the Polish space of all irreducible unitary representations of $G$ in some fixed separable Hilbert space of dimension $n$. Then the unitary dual is the quotient of the disjoint union $\bigsqcup \operatorname{Irr}_{n}(G)$ by the unitary equivalence relation, equipped with the corresponding quotient Borel structure. Recall that the unitary equivalence relation on $\bigsqcup \operatorname{Irr}_{n}(G)$ is said to be smooth if and only if the Mackey Borel structure on the unitary dual $\widehat{G}$ is countably separated. Of course, this is only problematic for the restriction of the unitary equivalence relation to $\operatorname{Irr}_{\infty}(G)$. The following result combines the main theorems of Glimm [21] and Thoma [46].

Theorem 1.1. If $G$ is a countable group, then the following conditions are equivalent:

(i) $G$ is not abelian-by-finite.

(ii) $G$ has an infinite dimensional irreducible representation.

(iii) The unitary equivalence relation on the space $\operatorname{Irr}_{\infty}(G)$ of infinite dimensional irreducible unitary representations of $G$ is not smooth.

Research partially supported by NSF Grant DMS 1101597. 
Furthermore, by Thoma [46], a countable group $G$ satisfies the conditions of Theorem 1.1 if and only if $G$ is a non-type $I$ group. ${ }^{1}$ While Theorem 1.1 shows that the equivalence classes of irreducible unitary representations of a countable non-type I group $G$ cannot be parameterized by the points of any Polish space, this is not enough in itself to rule out a satisfactory classification. For example, the isomorphism relation on the space of rank 1 torsion-free abelian groups is not smooth; and yet Baer [3] was able to provide a perfectly satisfactory classification. However, the following more recent result of Hjorth provides a much more serious obstruction to the existence of a satisfactory classification.

Theorem 1.2 (Hjorth [24]). If $G$ is a countable non-type I group, then there exists a $U(\mathcal{H})$-invariant Borel subset $X \subseteq \operatorname{Irr}_{\infty}(G)$ such that the action of $U(\mathcal{H})$ on $X$ is turbulent.

The notion of turbulence was introduced by Hjorth [25], in order to address the question of which orbit equivalence relations of continuous actions of Polish groups on Polish spaces are classifiable by countable structures. Here an analytic equivalence relation $E$ on a Polish space $X$ is said to admit classification by countable structures if there exists a Borel map $x \mapsto \mathcal{M}_{x}$ from $X$ to the space of countable structures for some countable language such that

$$
x E y \Longleftrightarrow \mathcal{M}_{x} \cong \mathcal{M}_{y} \text {. }
$$

For example, Farah-Toms-Tornquist [15] have verified that Elliott's classification [11] of the approximately finite dimensional $C^{*}$-algebras by $K$-theoretic invariants can be implemented in a Borel manner; and this means that the isomorphism relation for approximately finite dimensional $C^{*}$-algebras admits a classification by countable structures. On the other hand, Hjorth [25] has shown that if the continuous action of the Polish group $G$ on the Polish space $X$ is turbulent, then the corresponding orbit equivalence relation $E_{G}^{X}$ does not admit a classification by countable structures. In particular, if $G$ is a countable non-type I group, then

\footnotetext{
${ }^{1}$ The definition of a type I group can be found in Folland [17, Section 7.2]. This notion will play no role in this paper, other than to enable us to avoid repeatedly using the term "non-(abelianby-finite)"
} 
the unitary equivalence relation $\approx_{G}$ does not admit a classification by countable structures.

However, this is hopefully not the end of the story. While it does not seem feasible to find a satisfactory classification of the irreducible unitary representations of a fixed countable non-type I group, there remains the natural question of determining the relative complexities of the unitary duals of pairs $G, H$ of countable non-type I groups. For example, let $G$ be the direct sum of infinitely many copies of the symmetric group $\operatorname{Sym}(3)$ and let $\mathbb{F}_{\infty}$ be the free group on infinitely many generators. Then it seems reasonable to conjecture that the unitary dual of $\mathbb{F}_{\infty}$ is strictly more complex than that of $G$. In order to give a precise formulation of this question, it is first necessary to recall some of the basic notions from the theory of Borel equivalence relations.

If $X$ is a Polish space, then an equivalence relation $E$ on $X$ is said to be Borel if $E$ is a Borel subset of $X \times X$. For example, it is well-known that the unitary equivalence relation is an $F_{\sigma}$ equivalence relation on $\operatorname{Irr}_{n}(G)$. Suppose that $E, F$ are Borel equivalence relations on the Polish spaces $X, Y$ respectively. Then $E$ is Borel reducible to $F$, written $E \leq_{B} F$, if there exists a Borel map $f: X \rightarrow Y$ such that for all $x, z \in X$,

$$
x E z \Longleftrightarrow f(x) F f(z)
$$

$E$ and $F$ are Borel bireducible, written $E \sim_{B} F$, if both $E \leq_{B} F$ and $F \leq_{B} E$. Finally we write $E<_{B} F$ if both $E \leq_{B} F$ and $F \varliminf_{B} E$.

Here the idea is that Borel bireducibility captures the intuitive notion of two Borel equivalence relations having the same complexity. Some readers may be concerned that the Borel bireducibility of the unitary equivalence relations of the countable groups $G, H$ is strictly weaker than the usual notion in the literature of the Borel isomorphism of their unitary duals $\widehat{G}, \widehat{H}$. (For example, see Elliott [12].)

Definition 1.3. If $E, F$ are Borel equivalence relations on the Polish spaces $X, Y$ then the quotients $X / E, Y / F$ are Borel isomorphic, written $X / E \simeq Y / F$, if there exist Borel reductions $\varphi: X \rightarrow Y$ and $\psi: Y \rightarrow X$ such that the induced maps $\hat{\varphi}: X / E \rightarrow Y / F$ and $\hat{\psi}: Y / F \rightarrow X / E$ are mutually inverse bijections. 
However, since the unitary equivalence relation is the Borel orbit equivalence relation of a continuous action of a Polish group, the following result shows that these two notions coincide.

Theorem 1.4 (Motto Ros [39]). If E, F are Borel orbit equivalence relations arising from Borel actions of Polish groups on the Polish spaces $X, Y$, then $E \sim_{B} F$ if and only if $X / E \simeq Y / F$.

Of course, if $G$ is a countable group, then the unitary equivalence restricted to the space $\bigsqcup_{1 \leq n \in \mathbb{N}} \operatorname{Irr}_{n}(G)$ of finite dimensional irreducible unitary representations is smooth. Consequently, in this paper, our focus will be on the $\operatorname{space}^{\operatorname{Irr}_{\infty}}(G)$ of infinite dimensional irreducible unitary representations of $G$.

Definition 1.5. If $G$ is a countable non-type I group, then $\approx_{G}$ denotes the unitary equivalence relation on the space $\operatorname{Irr}_{\infty}(G)$ of infinite dimensional irreducible unitary representations of $G$.

In summary, the following result is an immediate consequence of Theorem 1.4.

Corollary 1.6. If $G, H$ are countable non-type I groups, then the following are equivalent:

(1) The unitary equivalence relations $\approx_{G}$ and $\approx_{H}$ are Borel bireducible.

(2) The unitary duals $\widehat{G}$ and $\widehat{H}$ are Borel isomorphic.

In the remainder of this section, we will discuss the main results of this paper. We will begin by considering the countable groups with the most complex unitary duals.

Definition 1.7. The countable group $G$ is representation universal if whenever $H$ is a countable group, then $\approx_{H}$ is Borel reducible to $\approx_{G}$

For example, the free group $\mathbb{F}_{\infty}$ on countably many generators is representation universal. To see this, let $G$ be any countable group and let $p: \mathbb{F}_{\infty} \rightarrow G$ be a surjective homomorphism. Then the map $\pi \mapsto \pi \circ p$ is clearly a Borel reduction from $\approx_{G}$ to $\approx_{\mathbb{F}_{\infty}}$. More generally, the same argument shows that if $G, H$ are countable groups and $G$ is a homomorphic image of $H$, then $\approx_{G}$ is Borel reducible to $\approx_{H}$. On the other hand, the following basic question remains open. 
Question 1.8. Suppose that $G, H$ are countable groups such that $G \leqslant H$. Does it necessarily follow that $\approx_{G}$ is Borel reducible to $\approx_{H}$ ?

In particular, while $\mathbb{F}_{\infty}$ is embeddable in $S L(3, \mathbb{Z})$, it is currently not known whether or not $S L(3, \mathbb{Z})$ is representation universal. On the other hand, the following result which we will prove in Section 3 implies that all countable nonabelian free groups are representation universal; and, of course, this means that very large countable groups are also representation universal. (Recall that a group $G$ is said to be very large if $G$ has a nonabelian free quotient.)

Theorem 1.9. The free group $\mathbb{F}_{2}$ on two generators is representation universal.

Very little is known concerning the closure properties of the class of representation universal groups. For example, it is not known whether or not a finite extension of a representation universal group is also representation universal; and, in particular, it is not known whether countable large groups are representation universal. (Recall that a group $G$ is said to be large if $G$ has a very large subgroup of finite index.)

In Sections 4 through 6, we will mainly focus on the unitary duals of countable amenable non-type I groups. Here our main result is the following theorem. (As we will explain, Theorem 1.10 is an easy consequence of results of Elliott [12] and Sutherland [45].)

Theorem 1.10. If $G$ is a countable non-type I group and $H$ is a countable amenable group, then $\approx_{H}$ is Borel reducible to $\approx_{G}$. In particular, if $G$ and $H$ are countable amenable non-type I groups, then $\approx_{G}$ and $\approx_{H}$ are Borel bireducible.

The main results of this paper can be summarized graphically by the following diagram. 
- Very large groups

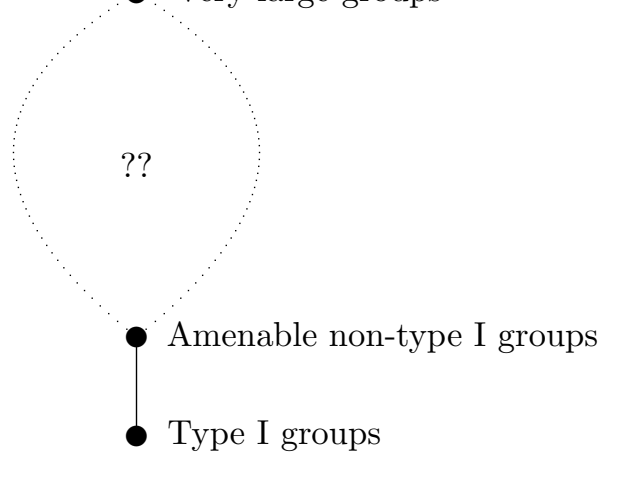

Here the dotted lines indicate that it is currently not known whether or not the unitary duals of all countable non-type I groups are Borel isomorphic. In Sections 6 and 7, we will present some evidence that suggests that countable amenable nontype I groups might not be representation universal. If this is indeed the case, then this raises the very interesting question of how many distinct unitary duals of countably infinite groups exist up to Borel isomorphism. Of course, ideally there should exist uncountably many such unitary duals. Unfortunately, the results of Section 5 suggest the possibility that perhaps the unitary duals of all countable non-amenable groups are Borel isomorphic.

Notation 1.11. If $\mathcal{H}$ is a (possibly finite dimensional) separable Hilbert space, then $B(\mathcal{H})$ denotes the space of bounded linear operators $T: \mathcal{H} \rightarrow \mathcal{H}$ and $U(\mathcal{H})$ denotes the corresponding unitary group.

Remark 1.12. For the convenience of the reader, the definitions of the various Borel equivalence relations that occur in this paper have been collected together in an Appendix.

Acknowledgements: I would like to thank Alexander Kechris for some very helpful discussions concerning the material in this paper and for explaining that Popa's Superrigidity Theorem [42] holds for cocycles taking values in the Banach space $\ell^{2}=\left\{\left(x_{n}\right) \in \mathbb{R}^{\mathbb{N}} \mid \sum x_{n}^{2}<\infty\right\}$ 


\section{Preliminaries}

In this section, we will recall some basic notions from the theory of countable Borel equivalence relations and from the representation theory of separable $C^{*}$ algebras. As expected, $C^{*}$-algebras will enter into our study through the canonical correspondence between the irreducible unitary representations of a countable group $G$ and the irreducible unitary representations of the associated group $C^{*}$-algebra $C^{*}(G)$. Countable Borel equivalence relations will enter the picture when we study the cocycles associated with the irreducible representations of semi-direct products of countable groups. (It is perhaps worth mentioning that Theorem 1.2 implies that if $G$ is a countable non-type I group, then the unitary equivalence relation $\approx_{G}$ is not Borel reducible to any countable Borel equivalence relation.)

2.1. Countable Borel Equivalence Relations and Ergodic theory. Let $X$ be a standard Borel space; i.e. a Polish space equipped with its associated $\sigma$-algebra of Borel subsets. Then the Borel equivalence relation $E$ on $X$ is said to be countable if every $E$-equivalence class is countable. For example, suppose that $G$ is a countable group and that $X$ is a standard Borel $G$-space; i.e. a standard Borel space $X$ equipped with a Borel action $(g, x) \mapsto g \cdot x$ of $G$ on $X$. Then the corresponding orbit equivalence relation $E_{G}^{X}$ is a countable Borel equivalence relation. Conversely, by Feldman-Moore [16], if $E$ is an arbitrary countable Borel equivalence relation on the standard Borel space $X$, then there exists a countable group $G$ and a Borel action of $G$ on $X$ such that $E=E_{G}^{X}$.

A detailed development of the general theory of countable Borel equivalence relations can be found in Jackson-Kechris-Louveau [28]. Here we will only recall those aspects of the theory that will play an essential role in this paper.

With respect to Borel reducibility, the least complex nonsmooth countable Borel equivalence relation is the Vitali equivalence relation $E_{0}$, which is defined on $2^{\mathbb{N}}$ by

$$
x E_{0} y \quad \Longleftrightarrow \quad x(n)=y(n) \quad \text { for almost all } n \text {. }
$$

More precisely, by Harrington-Kechris-Louveau [22], if $E$ is any (not necessarily countable) Borel equivalence relation, then $E$ is nonsmooth if and only if $E_{0} \leq_{B} E$. At the other extreme, let $E_{\infty}$ be the orbit equivalence relation arising from the shift 
action of the free group $\mathbb{F}_{2}$ on two generators on $2^{\mathbb{F}_{2}}$. Then, by Dougherty-JacksonKechris [10], $E_{\infty}$ is a universal countable Borel equivalence relation in the sense that $F \leq_{B} E_{\infty}$ for every countable Borel equivalence relation $F$.

If $X$ is a Polish space, then the Polish space of all Borel probability measures on $X$ is denoted by $\mathrm{P}(X)$. If $\mu, \nu \in \mathrm{P}(X)$, then $\mu$ and $\nu$ are said to be equivalent, written $\mu \sim \nu$, if for every Borel subset $A \subseteq X$,

$$
\mu(A)=0 \quad \Longleftrightarrow \quad \nu(A)=0 .
$$

If $G$ is a countable group and $X$ is a standard Borel $G$-space, then we can define a corresponding Borel action on $\mathrm{P}(X)$ by $\mu \stackrel{g}{\mapsto} g_{*} \mu$, where $g_{*} \mu$ is the Borel probability measure defined by

$$
g_{*} \mu(A)=\mu\left(g^{-1}(A)\right)
$$

for every Borel subset $A \subseteq X$. The Borel probability measure $\mu \in \mathrm{P}(X)$ is said to be invariant if $g_{*} \mu=\mu$ for all $g \in G$ and is said to be quasi-invariant if $g_{*} \mu \sim \mu$ for all $g \in G$. If $\mu \in \mathrm{P}(X)$ is quasi-invariant, then $\mu$ is said to be ergodic if every $G$-invariant Borel subset of $X$ is either null or conull.

Suppose that $E$ is a countable Borel equivalence relation on the standard Borel probability space $(X, \mu)$. Then $E$ is said to be measure-preserving if $\Gamma$ preserves $\mu$ for some (equivalently every) countable group $\Gamma$ acting in a Borel fashion such that $E=E_{\Gamma}^{X}$. In this case, $E$ is said to be ergodic if each such $\Gamma$ acts ergodically on $(X, \mu)$. If $E$ is an ergodic measure-preserving countable Borel equivalence relation on the standard Borel probability space $(X, \mu)$ and $A \subseteq X$ is a Borel subset such that $\mu(A)>0$, then $E \uparrow A$ denotes the restriction of $E$ to the standard Borel probability space $\left(A, \mu_{A}\right)$, where $\mu_{A}(C)=\mu(C) / \mu(A)$.

Suppose that $E, F$ are ergodic measure-preserving countable Borel equivalence relations on the standard Borel probability spaces $(X, \mu),(Y, \nu)$ respectively. Then $E$ and $F$ are said to be orbit equivalent if, after possibly deleting measure zero subsets, there exists a Borel bijection $\theta: X \rightarrow Y$ such that $\theta_{*} \mu=\nu$ and $\theta(E)=F$. More generally, $E$ and $F$ are said to be weakly orbit equivalent if there exist Borel subsets $A \subseteq X$ and $B \subseteq Y$ with $\mu(A), \nu(B)>0$ such that $E \uparrow A$ and $F \uparrow B$ are orbit equivalent. 
2.2. $C^{*}$-algebras. Let $\mathcal{A}$ be a separable $C^{*}$-algebra. For each $n \in\{1,2, \cdots, \infty\}$, let $\operatorname{Irr}_{n}(\mathcal{A})$ be the Polish space of all irreducible unitary representations of $\mathcal{A}$ in some fixed separable Hilbert space of dimension $n$. Then the spectrum $\widehat{\mathcal{A}}$ of $\mathcal{A}$ is the quotient of the disjoint union $\bigsqcup \operatorname{Irr}_{n}(\mathcal{A})$ by the unitary equivalence relation, equipped with the corresponding quotient Borel structure. By Glimm [21], the unitary equivalence relation on $\bigsqcup \operatorname{Irr}_{n}(\mathcal{A})$ is smooth if and only if $\mathcal{A}$ is a type I $C^{*}$ algebra. ${ }^{2}$ If $\mathcal{A}$ is a non-type I separable $C^{*}$-algebra, then $\approx_{\mathcal{A}}$ denotes the unitary

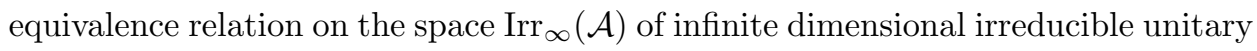
representations of $\mathcal{A}$.

Let $G$ be a countable group and let $\mathbb{C}[G]$ be the corresponding group algebra. Then the group $C^{*}$-algebra $C^{*}(G)$ is the completion of $\mathbb{C}[G]$ with respect to the norm

$$
\|x\|_{u}=\sup _{\pi}\|\pi(x)\|,
$$

where the supremum is taken over all cyclic $*$-representations $\pi: \mathbb{C}[G] \rightarrow B(\mathcal{H})$. It is well-known that there is a canonical correspondence between the irreducible unitary representations of $C^{*}(G)$ and the irreducible unitary representations of $G$. (For example, see Dixmier [9, Section 13.9].) Furthermore, this canonical correspondence witnesses that $\approx_{C^{*}(G)}$ and $\approx_{G}$ are Borel bireducible.

Remark 2.1. In 1967, Dixmier raised the question of whether the spectra of any two non-type I separable unital $C^{*}$-algebras are Borel isomorphic. In fact, this question is equivalent to asking whether countable amenable non-type I groups are representation universal. To see this, recall that if $\mathcal{A}$ is any separable unital $C^{*}$ algebra, then $\mathcal{A}$ is generated by a countable sequence of unitaries. It follows that there exists a surjective unital $*$-homomorphism $f: C^{*}\left(\mathbb{F}_{\infty}\right) \rightarrow \mathcal{A}$ and so

$$
\approx_{\mathcal{A}} \leq_{B} \approx_{C^{*}\left(\mathbb{F}_{\infty}\right)} \sim_{B} \approx_{\mathbb{F}_{\infty}}
$$

On the other hand, combining Theorem 4.2 and Corollary 6.2, it follows that if $G$ is a countable amenable non-type I group and $\mathcal{A}$ is any non-type I separable unital $C^{*}$-algebra, then

$$
\approx_{G} \sim_{B} \approx_{C^{*}(G)} \leq_{B} \approx_{\mathcal{A}}
$$

\footnotetext{
${ }^{2}$ The definition of a type I $C^{*}$-algebra can be found in Arveson [2]. Once again, this notion will play no role in this paper.
} 
Thus the following statements are equivalent:

- $\approx_{G}$ is Borel bireducible with $\approx_{\mathbb{F}_{\infty}}$ for some (equivalently every) countable amenable non-type I group $G$.

- $\widehat{\mathcal{A}}$ is Borel isomorphic with $\widehat{C^{*}\left(\mathbb{F}_{\infty}\right)}$ for every non-type I separable unital $C^{*}$-algebra $\mathcal{A}$.

\section{The Representation Universality of $\mathbb{F}_{2}$}

In this section, we will prove that the free group $\mathbb{F}_{2}$ on the two generators $\{a, b\}$ is representation universal. We have already noted that the free group $\mathbb{F}_{\infty}$ on infinitely many generators is representation universal. Thus it is enough to show that $\approx_{\mathbb{F}_{\infty}}$ is Borel reducible to $\approx_{\mathbb{F}_{2}}$.

Notation 3.1. If $H \leqslant G$ are countable groups and $\pi$ is a unitary representation of $H$, then $\operatorname{ind}_{H}^{G}(\pi)$ denotes the corresponding induced representation of $G$.

We will make use of the following well-known irreducibility criterion for representations induced from normal subgroups of countable groups. (For example, see the discussion in Quigg [43, Section 1].)

Definition 3.2. Suppose that $G$ is a countable group and that $N \unlhd G$ is a normal subgroup. If $\pi$ is a unitary representation of $N$ and $g \in G$, then $\pi^{g}$ denotes the unitary representation of $N$ defined by $\pi^{g}(x)=\pi\left(g x g^{-1}\right)$.

Theorem 3.3. Let $G$ be a countable group and let $N \unlhd G$ be a normal subgroup. If $\pi$ is an irreducible unitary representation of $N$, then the following are equivalent:

(i) $\operatorname{ind}_{N}^{G}(\pi)$ is an irreducible unitary representation of $G$.

(ii) $\pi^{g}$ is not unitarily equivalent to $\pi$ for all $g \in G \backslash N$.

Let $H=\operatorname{ker} h \unlhd \mathbb{F}_{2}$, where $h: \mathbb{F}_{2} \rightarrow \mathbb{Z}$ is the homomorphism such that $h(a)=1$ and $h(b)=0$. Then $T=\left\{a^{n} \mid n \in \mathbb{Z}\right\}$ is a set of coset representatives of $H$ in $\mathbb{F}_{2}$; and hence, by Schreier's Theorem, it follows that $S=\left\{a^{n} b a^{-n} \mid n \in \mathbb{Z}\right\}$ is a basis of $H$. (For example, see Serre [44, Proposition 16].) From now on, let $b_{n}=a^{n} b a^{-n}$, so that $a b_{n} a^{-1}=b_{n+1}$ for each $n \in \mathbb{Z}$. Let $f: \mathbb{N} \rightarrow \mathbb{N}$ be the function defined inductively by:

- $f(0)=0$; 
- $f(n+1)=1+\sum_{i=0}^{n} f(i)$;

and let $K=\{f(n) \mid n \in \mathbb{N}\}$. Then it is easily checked that $|(\ell+K) \cap K| \leq 1$ for all $0 \neq \ell \in \mathbb{Z}$. Hence, letting $c_{n}=b_{f(n)}$, the infinite subset $C=\left\{c_{n} \mid n \in \mathbb{N}\right\} \subseteq S$ satisfies $\left|a^{\ell} C a^{-\ell} \cap C\right| \leq 1$ for all $0 \neq \ell \in \mathbb{Z}$. From now on, we will identify $\mathbb{F}_{\infty}$ with the subgroup $\left\langle c_{n} \mid n \in \mathbb{N}\right\rangle$ of $H$ and we will identify $\operatorname{Irr}_{\infty}\left(\mathbb{F}_{\infty}\right)$ with the corresponding subspace of $\operatorname{Irr}_{\infty}(H)$; i.e. we will identify each $\pi \in \operatorname{Irr}_{\infty}\left(\mathbb{F}_{\infty}\right)$ with the corresponding representation $\pi \in \operatorname{Irr}_{\infty}(H)$ obtained by setting $\pi(s)=1$ for all $s \in S \backslash C$. Of course, with this identification, if $\pi, \sigma \in \operatorname{Irr}_{\infty}\left(\mathbb{F}_{\infty}\right)$, then

$$
\pi \approx_{\mathbb{F}_{\infty}} \sigma \Longleftrightarrow \pi \approx_{H} \sigma
$$

Let $\theta: \operatorname{Irr}_{\infty}\left(\mathbb{F}_{\infty}\right) \rightarrow \operatorname{Rep}\left(\mathbb{F}_{2}\right)$ be the Borel map defined by $\pi \mapsto \theta_{\pi}=\operatorname{ind}_{H}^{\mathbb{F}_{2}}(\pi)$. Then we will show that $\theta$ is a Borel reduction from $\approx_{\mathbb{F}_{\infty}}$ to $\approx_{\mathbb{F}_{2}}$.

Lemma 3.4. If $\pi \in \operatorname{Irr}_{\infty}\left(\mathbb{F}_{\infty}\right)$, then $\theta_{\pi} \in \operatorname{Irr}_{\infty}\left(\mathbb{F}_{2}\right)$.

Proof. By Theorem 3.3, it is enough to show that if $\ell \in \mathbb{Z} \backslash\{0\}$, then $\pi^{a^{\ell}}$ is not unitarily equivalent to $\pi$. So suppose that $\pi^{a^{\ell}}$ and $\pi$ are unitarily equivalent. Then since $a^{\ell} S a^{-\ell}=S$ and $\left|a^{\ell} C a^{-\ell} \cap C\right| \leq 1$, it follows that there exists at most one basis element $s \in S$ such that $\pi(s) \neq 1$. But this means that $\pi$ is a 1-dimensional representation of $H$, which is a contradiction.

Lemma 3.5. $\theta$ is a Borel reduction from $\approx_{\mathbb{F}_{\infty}}$ to $\approx_{\mathbb{F}_{2}}$.

Proof. First note that if $\pi, \sigma \in \operatorname{Irr}_{\infty}\left(\mathbb{F}_{\infty}\right)$ and $\pi \approx_{\mathbb{F}_{\infty}} \sigma$, then $\theta_{\pi} \approx_{\mathbb{F}_{2}} \theta_{\sigma}$. (For example, see Folland [17, Proposition 6.9].) Conversely, suppose that $\theta_{\pi} \approx_{\mathbb{F}_{2}} \theta_{\sigma}$. Then $\theta_{\pi} \uparrow H=\oplus_{\ell \in \mathbb{Z}} \pi^{a^{\ell}}$ and $\theta_{\sigma} \uparrow H=\oplus_{\ell \in \mathbb{Z}} \sigma^{a^{\ell}}$ are unitarily equivalent representations of $H$. Arguing as in the proof of Lemma 3.4, it follows that if $\ell \in \mathbb{Z} \backslash\{0\}$, then $\pi$ is not unitarily equivalent to $\sigma^{a^{\ell}}$. Hence $\pi \approx_{H} \sigma$ and so $\pi \approx_{\mathbb{F}_{\infty}} \sigma$.

\section{Representations of LOCALly Finite Groups}

In this section, we will derive the following weak form of Theorem 1.10 from a more general theorem of Elliott [12] on the Mackey Borel structures of the spectra of approximately finite-dimensional $C^{*}$-algebras.

Theorem 4.1. If $G$ is a countable non-type I group and $H$ is a countable locally finite group, then $\approx_{H}$ is Borel reducible to $\approx_{G}$. 
Recall that a $C^{*}$-algebra $\mathcal{A}$ is said to be approximately finite dimensional if $\mathcal{A}$ is the closure of the union $\bigcup_{n \in \mathbb{N}} \mathcal{A}_{n}$ of an increasing chain $\mathcal{A}_{0} \subseteq \mathcal{A}_{1} \subseteq \cdots \subseteq \mathcal{A}_{n} \subseteq \cdots$ of finite dimensional $C^{*}$-subalgebras. For example, let $H$ be a countable locally finite group and express $H=\bigcup_{n \in \mathbb{N}} H_{n}$ as the union of an increasing chain of finite subgroups $H_{n}$. Then the group $C^{*}$-algebra $C^{*}(H)$ is the closure of the group algebra $\mathbb{C}[H]=\bigcup_{n \in \mathbb{N}} \mathbb{C}\left[H_{n}\right]$ and hence $C^{*}(H)$ is approximately finite-dimensional. Also recall that if $G$ is any countable group, then $\approx_{C^{*}(G)}$ and $\approx_{G}$ are Borel bireducible. Consequently, Theorem 4.1 is an immediate consequence of the following result of Elliott [12].

Theorem 4.2 (Elliott [12]). If $\mathcal{A}$ is an approximately finite-dimensional $C^{*}$-algebra and $\mathcal{B}$ is a non-type I separable $C^{*}$-algebra, then $\approx_{\mathcal{A}}$ is Borel reducible to $\approx_{\mathcal{B}}$.

In the remainder of this section, we will point out a curious result concerning the asymptotic representation theory of finite groups, which follows easily from Thoma's Theorem [46], together with the theory of approximately finite-dimensional $C^{*}$ algebras.

Notation 4.3. If $K \leqslant L$ are finite groups and $\pi, \sigma$ are irreducible representations of $K, L$ respectively, then $\operatorname{mult}_{\pi}(\sigma \uparrow K)$ denotes the multiplicity with which $\pi$ occurs in the representation $\sigma \uparrow K$.

Theorem 4.4. If $G=\bigcup_{n \in \mathbb{N}} G_{n}$ is the union of the strictly increasing chain of finite subgroups $G_{n}$, then the following statements are equivalent:

(i) $G$ is not abelian-by-finite.

(ii) There exists a subsequence $\left(\ell_{n} \mid n \in \mathbb{N}\right)$ and irreducible representations $\pi_{n} \in \operatorname{Irr}\left(G_{\ell_{n}}\right)$ such that for all $n \in \mathbb{N}$, mult $_{\pi_{n}}\left(\pi_{n+1} \uparrow G_{\ell_{n}}\right)>1$.

Proof. To see that (ii) implies (i), suppose that $G$ contains an abelian subgroup $A$ such that $[G: A]=d<\infty$. Then $A_{n}=G_{n} \cap A$ is an abelian subgroup of $G_{n}$ such that $\left[G_{n}: A_{n}\right] \leq d$; and a simple application of the Frobenius reciprocity theorem shows that $\operatorname{deg} \pi \leq\left[G_{n}: A_{n}\right]$ for all $\pi \in \operatorname{Irr}\left(G_{n}\right)$. (For example, see Remark 17.12 in Huppert [27].) Of course, if $n<m$ and $\pi \in \operatorname{Irr}\left(G_{n}\right), \chi \in \operatorname{Irr}\left(G_{m}\right)$ satisfy $\operatorname{mult}_{\pi}\left(\chi \uparrow G_{n}\right)>1$, then $\operatorname{deg} \chi>\operatorname{deg} \pi$. Hence statement (ii) fails.

Conversely, suppose that $G$ is not abelian-by-finite. Then, by Thoma [46], $G$ is not of type $\mathrm{I}$ and so the corresponding group $C^{*}$-algebra $C^{*}(G)$ is a non-type 
I approximately finite-dimensional $C^{*}$-algebra. Notice that the Bratteli diagram associated with the increasing chain

$$
\mathbb{C}\left[G_{0}\right] \subseteq \mathbb{C}\left[G_{1}\right] \subseteq \cdots \subseteq \mathbb{C}\left[G_{n}\right] \subseteq \cdots
$$

of finite dimensional $C^{*}$-subalgebras is precisely the branching diagram of the irreducible representations of the groups $G_{n}$ in the corresponding increasing chain

$$
G_{0} \subseteq G_{1} \subseteq \cdots \subseteq G_{n} \subseteq \cdots
$$

of finite subgroups of $G$. Thus statement (ii) is immediate consequence of the Lazar-Taylor characterization [37, Theorem 3.13] of type I approximately finitedimensional $C^{*}$-algebras in terms of the multiplicities of paths through their Bratteli diagrams.

Question 4.5. Is there an "elementary" proof of Theorem 4.4? In other words, is Theorem 4.4 a consequence of some result concerning the branching diagrams of pairs $K \leqslant L$ of finite groups?

\section{Cocycles And Representations}

Suppose that $G=A \rtimes \Gamma$ is a semidirect product of the countable groups $A$ and $\Gamma$, where $A$ is an infinite abelian group. Then it is well-known that the irreducible representations of $G$ are determined by the $\Gamma$-quasi-invariant ergodic measures on the unitary dual $\widehat{A}$, together with the "irreducible" cocycles for the dual action of $\Gamma$ on $\widehat{A}$. Furthermore, the unitary equivalence relation $\approx_{G}$ is determined by:

- the measure equivalence relation on the space of $\Gamma$-quasi-invariant ergodic measures; together with

- the cocycle equivalence relation on the space of "irreducible" cocycles.

In his remarkable paper [45], Sutherland proved that if $\Gamma$ is amenable and acts freely and ergodically on $\widehat{A}$, then the unitary equivalence relation $\approx_{H}$ of every countable amenable group $H$ is Borel reducible to the cocycle equivalence relation on the space of "irreducible" cocycles $\sigma: \Gamma \times \widehat{A} \rightarrow U(\mathcal{H})$, where $\mathcal{H}$ is an infinite dimensional separable Hilbert space. As we will see, in combination with Elliott's work [12], this easily implies that the unitary duals of any two countable amenable non-type I groups are Borel isomorphic. In this section, we will begin our account 
of Sutherland's work, slightly expanded and generalized so that it is also applicable to non-amenable groups.

If $G$ is a countable group and $\mathcal{H}$ is a (possibly finite dimensional) separable Hilbert space, then $\operatorname{Rep}(G, \mathcal{H})$ denotes the Polish space of all unitary representations of $G$ in $\mathcal{H}$ and $\operatorname{Irr}(G, \mathcal{H})$ denotes the subspace of irreducible representations. For each pair $\pi, \sigma \in \operatorname{Rep}(G, \mathcal{H})$, let

$$
\mathcal{C}_{G}(\pi, \sigma)=\{S \in B(\mathcal{H}) \mid S \circ \pi(g)=\sigma(g) \circ S \text { for all } g \in G\},
$$

Recall that, by Schur's Lemma, if $\pi \in \operatorname{Rep}(G, \mathcal{H})$, then $\pi$ is irreducible if and only if $\mathcal{C}_{G}(\pi, \pi)$ is the vector space of scalar multiples of the identity operator. Furthermore, if $\pi, \sigma \in \operatorname{Rep}(G, \mathcal{H})$ are both irreducible and $0 \neq T \in \mathcal{C}_{G}(\pi, \sigma)$, then $T$ is a non-zero scalar multiple of a unitary operator and so $\pi, \sigma$ are unitarily equivalent.

We are now ready to begin our discussion of cocycles and the associated unitary representations. Suppose that the countable group $\Gamma$ acts freely and ergodically via measure-preserving Borel maps on the standard probability space $(X, \mu)$. Then a Borel map $\sigma: \Gamma \times X \rightarrow U(\mathcal{H})$ is said to be a cocycle if for all $\gamma, \gamma^{\prime} \in \Gamma$,

$$
\sigma\left(\gamma \gamma^{\prime}, x\right)=\sigma\left(\gamma, \gamma^{\prime} \cdot x\right) \sigma\left(\gamma^{\prime}, x\right) \quad \mu \text {-a.e. } x \in X \text {. }
$$

Let $Z^{1}(\Gamma \times X, U(\mathcal{H}))$ be the standard Borel space of Borel cocycles, where we identify two such maps $\sigma, \sigma^{\prime}$ if for all $\gamma \in \Gamma$,

$$
\sigma(\gamma, x)=\sigma^{\prime}(\gamma, x) \quad \mu \text {-a.e. } x \in X .
$$

Then for each cocycle $\sigma \in Z^{1}(\Gamma \times X, U(\mathcal{H}))$, we can define a corresponding unitary representation $\pi_{\sigma}$ of $\Gamma$ on the Hilbert space $L^{2}(X, \mathcal{H})$ by setting

$$
\left(\pi_{\sigma}(\gamma) \cdot f\right)(x)=\sigma\left(\gamma^{-1}, x\right)^{-1} f\left(\gamma^{-1} \cdot x\right)
$$

for each $\gamma \in \Gamma, x \in X$ and $f \in L^{2}(X, \mathcal{H})$.

Definition 5.1 (Sutherland [45]). If $\sigma, \tau \in Z^{1}(\Gamma \times X, U(\mathcal{H}))$, then $\operatorname{Hom}_{\Gamma}(\sigma, \tau)$ consists of the bounded Borel maps $T: X \rightarrow B(\mathcal{H})$ such that for all $\gamma \in \Gamma$,

$$
\sigma(\gamma, x) T(x)=T(\gamma \cdot x) \tau(\gamma, x) \quad \mu \text {-a.e. } x \in X,
$$

where we again identify two such maps if they agree $\mu$-a.e. 


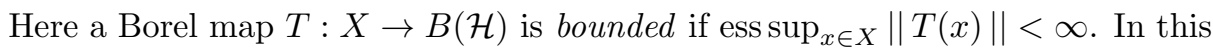
case, we can define a corresponding bounded linear operator $\int^{\oplus} T=\int^{\oplus} T(x) d \mu(x)$ on $L^{2}(X, \mathcal{H})$ by $\left[\left(\int^{\oplus} T\right) f\right](x)=T(x) f(x)$.

Lemma 5.2. If $\sigma, \tau \in Z^{1}(\Gamma \times X, U(\mathcal{H}))$ and $T: X \rightarrow B(\mathcal{H})$ is a bounded Borel map, then $T \in \operatorname{Hom}_{\Gamma}(\sigma, \tau)$ if and only if $\int^{\oplus} T \in \mathcal{C}_{\Gamma}\left(\pi_{\sigma}, \pi_{\tau}\right)$.

Proof. A routine calculation.

Next, by passing from $\Gamma$ to a suitable semidirect product $G=A \rtimes \Gamma$ and then extending each $\pi_{\sigma}$ to a corresponding unitary representation of $G$, we will ensure that for every $S \in \mathcal{C}_{G}\left(\pi_{\sigma}, \pi_{\tau}\right)$, there exists a bounded Borel map $T: X \rightarrow B(\mathcal{H})$ such that $S=\int^{\oplus} T$. In more detail, suppose that $A$ is a countably infinite abelian group and that $\gamma \mapsto \varphi_{\gamma}$ is a homomorphism from $\Gamma$ to $\operatorname{Aut}(A)$. Then we can define an induced action of $\Gamma$ on the unitary dual $X=\operatorname{Irr}_{1}(A)$ of $A$ by $\gamma \cdot x=x \circ \varphi_{\gamma}^{-1}$. Next suppose that $\mu$ is a $\Gamma$-invariant probability measure on $X$ such that the action of $\Gamma$ on $(X, \mu)$ is essentially free and ergodic. Let $G=A \rtimes_{\varphi} \Gamma$ be the corresponding semi-direct product defined by

$$
(a, \gamma)\left(a^{\prime}, \gamma^{\prime}\right)=\left(a \varphi_{\gamma}\left(a^{\prime}\right), \gamma \gamma^{\prime}\right)
$$

Then for each cocycle $\sigma \in Z^{1}(\Gamma \times X, U(\mathcal{H}))$, we can extend the unitary representation $\pi_{\sigma}$ of $\Gamma$ on $L^{2}(X, \mathcal{H})$ to a unitary representation of $G=A \rtimes_{\varphi} \Gamma$ by setting

$$
\left(\pi_{\sigma}(a) \cdot f\right)(x)=x(a) f(x)
$$

Notice that the direct integral decomposition of $\pi_{0}=\pi_{\sigma} \uparrow A$ as $\pi_{0}=\int^{\oplus} x d \mu(x)$ on $L^{2}(X, \mathcal{H})=\int^{\oplus} \mathcal{H}_{x} d \mu(x)$, where each $\mathcal{H}_{x}=\mathcal{H}$, is the canonical direct integral decomposition of $\pi_{0}$ into primary representations. It follows that if $S \in \mathcal{C}_{A}\left(\pi_{0}, \pi_{0}\right)$, then there exists a bounded Borel map $T: X \rightarrow B(\mathcal{H})$ such that $S=\int^{\oplus} T$. (For example, see Nielsen [40].)

Proposition 5.3. With the above hypotheses, if $\sigma, \tau \in Z^{1}(\Gamma \times X, U(\mathcal{H}))$, then the map $T \mapsto \int^{\oplus} T$ is an vector space isomorphism between $\operatorname{Hom}_{\Gamma}(\sigma, \tau)$ and $\mathcal{C}_{G}\left(\pi_{\sigma}, \pi_{\tau}\right)$.

Proof. If $S \in \mathcal{C}_{G}\left(\pi_{\sigma}, \pi_{\tau}\right)$, then $S \in \mathcal{C}_{A}\left(\pi_{0}, \pi_{0}\right)$, where $\pi_{0}=\pi_{\sigma} \uparrow A=\pi_{\tau}$, and so there exists a bounded Borel map $T: X \rightarrow B(\mathcal{H})$ such that $S=\int^{\oplus} T$. Hence the result follows from Lemma 5.2. 
In particular, by Schur's Lemma, if $\sigma \in Z^{1}(\Gamma \times X, U(\mathcal{H}))$, then the corresponding unitary representation $\pi_{\sigma}$ of $G=A \rtimes \Gamma$ is irreducible if and only if $\operatorname{Hom}_{\Gamma}(\sigma, \sigma)$ consists of the constant $\mu$-a.e. maps $T: X \rightarrow B(\mathcal{H})$ taking values in the vector space of scalar multiples of the identity. In this case, we say that $\sigma$ is an irreducible cocycle. Let $\operatorname{Irr}(\Gamma \times X, U(\mathcal{H})) \subseteq Z^{1}(\Gamma \times X, U(\mathcal{H}))$ be the set of irreducible cocycles. Since $\operatorname{Irr}(\Gamma \times X, U(\mathcal{H}))$ is the inverse image of the set of irreducible representations under the Borel map $\sigma \mapsto \pi_{\sigma}$, it follows that $\operatorname{Irr}(\Gamma \times X, U(\mathcal{H}))$ is a Borel subset of $Z^{1}(\Gamma \times X, U(\mathcal{H}))$ and hence $\operatorname{Irr}(\Gamma \times X, U(\mathcal{H}))$ is a standard Borel space.

Next suppose that $\sigma, \tau \in \operatorname{Irr}(\Gamma \times X, U(\mathcal{H}))$ are irreducible cocycles. Then Proposition 5.3 implies that $\pi_{\sigma}$ and $\pi_{\tau}$ are unitarily equivalent if and only if there exists $0 \neq T \in \operatorname{Hom}_{\Gamma}(\sigma, \tau)$ such that $S=\int^{\oplus} T$ satisfies

$$
S \circ \pi_{\sigma}(g)=\pi_{\tau}(g) \circ S \quad \text { for all } g \in G
$$

Furthermore, in this case, once again by Schur's Lemma, $S$ is a nonzero scalar multiple of a unitary operator. Hence, replacing $T$ by a suitable scalar multiple, we can assume that $S$ is a unitary operator. By Nielsen [40, Proposition 6.1(d)], it follows that $T(x) \in U(\mathcal{H})$ for $\mu$-a.e. $x \in X$ and so we can regard $T$ as an element of the Polish group $L(X, U(\mathcal{H}))$. Here $L(X, U(\mathcal{H}))$ is the space of Borel maps $T: X \rightarrow U(\mathcal{H})$ equipped with the group operation of pointwise multiplication, where we identify two such maps if they agree $\mu$-a.e. (For more details, see Kechris [33, Section 19].) The group $L(X, U(\mathcal{H}))$ acts on $Z^{1}(\Gamma \times X, U(\mathcal{H}))$ by

$$
(T \cdot \sigma)(\gamma, x)=T(\gamma \cdot x) \sigma(\gamma, x) T(x)^{-1}
$$

and it is easily checked that $\operatorname{Irr}(\Gamma \times X, U(\mathcal{H}))$ is invariant under this action. Summing up, we now see that the map $\sigma \mapsto \pi_{\sigma}$ is a Borel reduction from the orbit equivalence relation for the action of $L(X, U(\mathcal{H}))$ on $\operatorname{Irr}(\Gamma \times X, U(\mathcal{H}))$ to the unitary equivalence relation on $\operatorname{Irr}\left(G, L^{2}(X, U(\mathcal{H}))\right)$.

Conversely, the following result will enable us to reduce the unitary equivalence relations $\approx_{H}$ for various countable groups $H$ to suitable cocycle equivalence relations.

Proposition 5.4. Let $\Gamma, H$ be countable groups and let $\rho: \Gamma \rightarrow H$ be a surjective homomorphism. Suppose that: 
(i) $\Gamma$ acts freely and ergodically via measure-preserving Borel maps on the standard probability space $(X, \mu)$.

(ii) $\operatorname{ker} \rho$ acts ergodically on $(X, \mu)$.

Let $\pi \mapsto \sigma_{\pi}$ be the Borel map from $\operatorname{Rep}(H, \mathcal{H})$ to $Z^{1}(\Gamma \times X, U(\mathcal{H}))$ defined by

$$
\sigma_{\pi}(\gamma, x)=(\pi \circ \rho)(\gamma) \quad \gamma \in \Gamma, x \in X
$$

If $\sigma, \theta \in \operatorname{Rep}(H, \mathcal{H})$, then $\operatorname{Hom}_{\Gamma}\left(\sigma_{\pi}, \sigma_{\theta}\right)$ is the vector space of constant maps taking values in $\mathcal{C}_{H}(\pi, \theta)$.

Proof. Clearly if $T \in \mathcal{C}_{H}(\pi, \theta)$, then the constant map $x \mapsto T$ lies in $\operatorname{Hom}_{\Gamma}\left(\sigma_{\pi}, \sigma_{\theta}\right)$. Conversely, if $T \in \operatorname{Hom}_{\Gamma}\left(\sigma_{\pi}, \sigma_{\theta}\right)$, then for all $\gamma \in \Gamma$,

$$
\sigma_{\pi}(\gamma, x) T(x)=T(\gamma \cdot x) \sigma_{\theta}(\gamma, x) \quad \mu \text {-a.e. } x \in X
$$

In particular, for each $\gamma \in \operatorname{ker} \rho$, we have that

$$
T(x)=T(\gamma \cdot x) \quad \mu \text {-a.e. } x \in X
$$

Since ker $\rho$ acts ergodically on $X$, it follows that $T$ is $\mu$-a.e. constant; and since the homomorphism $\rho: \Gamma \rightarrow H$ is surjective, it follows that the $\mu$-a.e. constant value of $T$ lies in $\mathcal{C}_{H}(\pi, \theta)$.

Following Kechris [33, Section 20(B)], we can reformulate the notion of a cocycle for an arbitrary countable ergodic measure-preserving equivalence relation $E$ on the standard Borel probability space $(X, \mu)$ as follows. First let $M$ be the $\sigma$-finite Borel measure defined on $E$ by

$$
M(A)=\int\left|A_{x}\right| d \mu(x)
$$

where $A_{x}=\{y \in X \mid(x, y) \in A\}$. (For example, see Kechris [33, Section 6.B].) Then $Z^{1}(E, U(\mathcal{H}))$ denotes the standard Borel space of Borel maps $\sigma: E \rightarrow U(\mathcal{H})$ such that

$$
\sigma(x, z)=\sigma(y, z) \sigma(x, y)
$$

for all $x E y E z$ in some $E$-invariant Borel subset of $X$ of $\mu$-measure 1, where we identify two such maps $\sigma, \sigma^{\prime}$ if

$$
\sigma(x, z)=\sigma^{\prime}(x, z) \quad M \text {-a.e. }(x, z) \in E .
$$


If $\sigma, \tau \in Z^{1}(E, U(\mathcal{H}))$, then we define $\operatorname{Hom}_{E}(\sigma, \tau)$ to consist of the bounded Borel maps $T: X \rightarrow B(\mathcal{H})$ such that

$$
\sigma(x, z) T(x)=T(z) \tau(x, z) \quad M \text {-a.e. }(x, z) \in E,
$$

where we identify two such maps if they agree $\mu$-a.e.

Of course, if $E=E_{\Gamma}^{X}$ is the orbit equivalence relation arising from a free ergodic measure-preserving action on $(X, \mu)$, then for each $\sigma \in Z^{1}(\Gamma \times X, U(\mathcal{H}))$, we can define a corresponding cocycle $\sigma^{\prime} \in Z^{1}(E, U(\mathcal{H}))$ by

$$
\sigma^{\prime}(x, z)=\sigma(g, x)
$$

where $g \in \Gamma$ is the unique $g \in \Gamma$ such that $g \cdot x=z$; and for each $\tau \in Z^{1}(E, U(\mathcal{H}))$, we can define a corresponding cocycle $\tau^{\prime} \in Z^{1}(\Gamma \times X, U(\mathcal{H}))$ by

$$
\tau^{\prime}(g, x)=\tau(x, g \cdot x)
$$

Notice that if $\sigma_{1}, \sigma_{2} \in Z^{1}(\Gamma \times X, U(\mathcal{H}))$ and $\sigma_{1}^{\prime}, \sigma_{2}^{\prime} \in Z^{1}(E, U(\mathcal{H}))$ are the corresponding cocycles as above, then for all bounded Borel maps $T: X \rightarrow B(\mathcal{H})$,

$$
T \in \operatorname{Hom}_{\Gamma}\left(\sigma_{1}, \sigma_{2}\right) \Longleftrightarrow T \in \operatorname{Hom}_{E}\left(\sigma_{1}^{\prime}, \sigma_{2}^{\prime}\right) .
$$

Definition 5.5. A cocycle $\sigma \in Z^{1}(E, U(\mathcal{H}))$ is said to be irreducible if $\operatorname{Hom}_{E}(\sigma, \sigma)$ consists of the constant $\mu$-a.e. maps $T: X \rightarrow B(\mathcal{H})$ taking values in the vector space of scalar multiples of the identity.

For each (possibly finite dimensional) separable Hilbert space $\mathcal{H}$, let $\operatorname{Irr}(E, U(\mathcal{H})$ ) be the set of irreducible cocycles.

Definition 5.6. Let $\mathcal{H}$ be an infinite dimensional separable Hilbert space. Then $\equiv_{E}$ denotes the orbit equivalence relation arising from the action of $L(X, U(\mathcal{H}))$ on $\operatorname{Irr}(E, U(\mathcal{H}))$ defined by

$$
(f \cdot \sigma)(x, z)=f(z) \sigma(x, z) f(x)^{-1}
$$

for each $f \in L(X, U(\mathcal{H}))$ and $\sigma \in \operatorname{Irr}(E, U(\mathcal{H}))$.

If $E=E_{\Gamma}^{X}$, then we will often identify $Z^{1}(E, U(\mathcal{H}))$ with $Z^{1}(\Gamma \times X, U(\mathcal{H}))$ and $\operatorname{Irr}(E, U(\mathcal{H}))$ with $\operatorname{Irr}(\Gamma \times X, U(\mathcal{H}))$.

It is well-known that if two ergodic measure-preserving actions of countable groups are orbit equivalent, then the associated cocycle machineries are isomorphic. 
(See Feldman-Moore [16].) In fact, as observed by Kechris [33, Section 20(G)], the same is true when the actions are weakly orbit equivalent.

Lemma 5.7. Suppose that $E$ and $F$ are countable ergodic measure-preserving equivalence relations on the standard Borel probability spaces $(X, \mu),(Y, \nu)$ respectively. If $E$ and $F$ are weakly orbit equivalent, then $\equiv_{E}$ and $\equiv_{F}$ are Borel bireducible.

Proof. It is enough to prove Lemma 5.7 for the special case where $(Y, \nu)=\left(A, \mu_{A}\right)$ for some Borel a.e.-complete section $A \subseteq X$; i.e. $A$ is a Borel subset which meets $\mu$-a.e. $E$-class and $\mu_{A}$ is the Borel probability measure defined on $A$ by $\mu_{A}(Z)=$ $\mu(Z) / \mu(A)$. For each $\sigma \in Z^{1}(E, U(\mathcal{H}))$, let $\sigma \uparrow A \in Z^{1}(E \uparrow A, U(\mathcal{H}))$ be the restriction of $\sigma$ to $A$. By Kechris [33, Section 20(G)], if $\sigma_{1}, \sigma_{2} \in Z^{1}(E, U(\mathcal{H}))$, then

$$
\sigma_{1} \equiv_{E} \sigma_{2} \Longleftrightarrow \sigma_{1}\left\lceil A \equiv_{E \uparrow A} \quad \sigma_{2} \uparrow A .\right.
$$

Next let $f_{A}: X \rightarrow A$ be a Borel map such that $f_{A}(x) E x$ for $\mu$-a.e. $x \in X$ and such that $f_{A} \backslash A=\operatorname{Id}_{A}$. Then for each $\tau \in Z^{1}(E \uparrow A, U(\mathcal{H}))$, we can define a corresponding cocycle $\tau^{A} \in Z^{1}(E, U(\mathcal{H}))$ by $\tau^{A}(x, z)=\tau\left(f_{A}(x), f_{A}(z)\right)$. It is easily seen that if $\tau_{1}, \tau_{2} \in Z^{1}(E \uparrow A, U(\mathcal{H}))$, then

$$
\tau_{1} \equiv_{E \uparrow A} \quad \tau_{2} \quad \Longleftrightarrow \quad \tau_{1}^{A} \equiv_{E} \tau_{2}^{A} .
$$

Furthermore, by Kechris [33, Section $20(\mathrm{G})]$, for each $\sigma \in Z^{1}(E, U(\mathcal{H}))$,

$$
\sigma \equiv_{E}(\sigma \uparrow A)^{A} .
$$

Thus it is enough to show that if $\tau \in Z^{1}(E\lceil A, U(\mathcal{H}))$, then

$$
\tau \in \operatorname{Irr}\left(E\lceil A, U(\mathcal{H})) \quad \Longleftrightarrow \quad \tau^{A} \in \operatorname{Irr}(E, U(\mathcal{H})) .\right.
$$

First suppose that $\tau \notin \operatorname{Irr}\left(E\lceil A, U(\mathcal{H}))\right.$. Then there exists $T \in \operatorname{Hom}_{E\lceil A}(\tau, \tau)$ such that $T$ is not a scalar multiple of the identity $\nu$-a.e. Let $T^{A}: X \rightarrow B(\mathcal{H})$ be the bounded Borel map defined by $T^{A}(x)=T\left(f_{A}(x)\right)$. Then $T^{A} \in \operatorname{Hom}_{E}\left(\tau^{A}, \tau^{A}\right)$ witnesses that $\tau^{A} \notin \operatorname{Irr}(E, U(\mathcal{H}))$. Conversely, suppose that $\tau \in \operatorname{Irr}(E \uparrow A, U(\mathcal{H}))$ and let $T \in \operatorname{Hom}_{E}\left(\tau^{A}, \tau^{A}\right)$. Then

$$
\tau(x, z) T(x)=T(z) \tau(x, z) \quad M \text {-a.e. }(x, z) \in E \uparrow A,
$$

and hence there exists $\lambda \in \mathbb{C}$ such that $T(x)=\lambda \operatorname{Id}_{\mathcal{H}}$ for $\mu$-a.e. $x \in A$. Since

$$
\tau^{A}\left(x, f_{A}(x)\right) T(x)=T\left(f_{A}(x)\right) \tau^{A}\left(x, f_{A}(x)\right) \quad \mu \text {-a.e. } x \in X,
$$


it follows that $T(x)=\lambda \operatorname{Id}_{\mathcal{H}}$ for $\mu$-a.e. $x \in X$. Thus $\tau^{A} \in \operatorname{Irr}(E, U(\mathcal{H}))$.

In the next section, following Sutherland [45], we will use the flexibility inherent in Lemma 5.7 to compare the unitary duals of countable amenable non-type I groups. For now, we will just record the following result.

Theorem 5.8. $\approx_{\mathbb{F}_{2}}$ is Borel bireducible with $\equiv_{E_{\infty}}$.

Proof. In order to see that $\equiv_{E_{\infty}}$ is Borel reducible to $\approx_{\mathbb{F}_{2}}$, let $\mu$ is the usual product probability measure on $2^{\mathbb{F}_{2}}$ and identify $\operatorname{Irr}\left(E_{\infty}, U(\mathcal{H})\right)$ with $\operatorname{Irr}\left(\mathbb{F}_{2} \times 2^{\mathbb{F}_{2}}, U(\mathcal{H})\right)$. Let $C_{2}$ be the cyclic group of order 2 and let

$$
G=C_{2} \operatorname{wr} \mathbb{F}_{2}=A \rtimes \mathbb{F}_{2},
$$

where $A$ is the base group of the wreath product. Then the induced action of $\mathbb{F}_{2}$ on the unitary dual of $A$ is isomorphic to the shift action of $\mathbb{F}_{2}$ on $\left(2^{\mathbb{F}_{2}}, \mu\right)$. Consider the Borel map $\sigma \mapsto \pi_{\sigma}$ from $Z^{1}\left(\mathbb{F}_{2} \times 2^{\mathbb{F}_{2}}, U(\mathcal{H})\right)$ to $\operatorname{Rep}\left(G, L^{2}\left(2^{\mathbb{F}_{2}}, \mathcal{H}\right)\right)$ defined by equations (5.2) and (5.3). Applying Proposition 5.3 and Schur's Lemma,

(i) $\operatorname{Irr}\left(\mathbb{F}_{2} \times 2^{\mathbb{F}_{2}}, U(\mathcal{H})\right)$ is mapped to $\operatorname{Irr}\left(G, L^{2}\left(2^{\mathbb{F}_{2}}, \mathcal{H}\right)\right)$; and

(ii) if $\sigma, \tau \in \operatorname{Irr}\left(\mathbb{F}_{2} \times 2^{\mathbb{F}_{2}}, U(\mathcal{H})\right)$, then $\sigma \equiv_{E_{\infty}} \tau$ if and only if $\pi_{\sigma} \approx_{G} \pi_{\tau}$.

Thus $\equiv_{E_{\infty}}$ is Borel reducible to $\approx_{G}$; and applying Theorem 1.9, it follows that $\equiv_{E_{\infty}}$ is Borel reducible to $\approx_{\mathbb{F}_{2}}$.

In order to see that $\approx_{\mathbb{F}_{2}}$ is Borel reducible to $\equiv_{E_{\infty}}$, let $\nu$ be the usual product probability measure on $2^{\mathbb{F}_{3}}$ and consider the shift action of $\mathbb{F}_{3}$ on $\left(2^{\mathbb{F}_{3}}, \nu\right)$. Let $\rho: \mathbb{F}_{3} \rightarrow \mathbb{F}_{2}$ be a surjective homomorphism. Since the shift action of $\mathbb{F}_{3}$ on $\left(2^{\mathbb{F}_{3}}, \nu\right)$ is strongly mixing, it follows that $\operatorname{ker} \rho$ acts ergodically on $\left(2^{\mathbb{F}_{3}}, \nu\right)$. It follows that the Borel map $\pi \mapsto \sigma_{\pi}$ from $\operatorname{Rep}\left(\mathbb{F}_{2}, \mathcal{H}\right)$ to $Z^{1}\left(\mathbb{F}_{3} \times 2^{\mathbb{F}_{3}}, U(\mathcal{H})\right)$ given by Proposition 5.4 induces a Borel reduction from $\approx_{\mathbb{F}_{2}}$ to $\equiv_{E}$, where $E=E_{\mathbb{F}_{3}}^{2^{\mathbb{F}_{3}}}$. By Bowen [5], $E$ is weakly orbit equivalent with $E_{\infty}=E_{\mathbb{F}_{2}}^{\mathbb{R}^{\mathbb{F}_{2}}}$; and hence, by Lemma 5.7, $\equiv_{E}$ is Borel bireducible with $\equiv_{E_{\infty}}$.

Remark 5.9. As we mentioned earlier, it is currently not known whether every countable group containing a nonabelian free subgroup is representation universal. On the other hand, the methods of this section, together with the "measurablegroup-theoretical solution to von Neumann's problem" of Gaboriau-Lyons [18], 
suggest the possibility that perhaps every countable non-amenable group is representation universal. (Cf. Epstein [13].)

\section{Representations of amenable groups}

In the first part of this section, combining the results of Elliott [12] and Sutherland [45], we will prove that if $G$ and $H$ are countable amenable non-type I groups, then the unitary equivalence relations $\approx_{G}$ and $\approx_{H}$ are Borel bireducible. Then, in the remainder of this section, we will discuss a possible (albeit very optimistic) strategy for showing that countable amenable non-type I groups are not representation universal.

Theorem 6.1. If $H$ is a countable amenable non-type I group, then $\approx_{H}$ is Borel bireducible with $\equiv_{E_{0}}$.

Corollary 6.2. If $G$ and $H$ are countable amenable non-type I groups, then $\approx_{G}$ and $\approx_{H}$ are Borel bireducible.

Corollary 6.3. If $G$ is a countable non-type $I$ group and $H$ is any countable amenable group, then $\approx_{H}$ is Borel reducible to $\approx_{G}$.

Proof. If $H$ is a type I group, then $\approx_{H}$ is smooth and hence $\approx_{H}$ is Borel reducible to $\approx_{G}$. Thus we can suppose that $H$ is a countable amenable non-type I group. Let $K$ be a countable locally finite non-type I group. Then by Theorem $6.1, \approx_{H}$ and $\approx_{K}$ are Borel bireducible; and by Theorem $4.1, \approx_{K}$ is Borel reducible to $\approx_{G}$.

It should be stressed that the following is essentially just a slight reformulation of the argument in Sutherland [45]. In particular, the key idea of using the OrnsteinWeiss Theorem in this setting is due to Sutherland.

Proof of Theorem 6.1. To see that $\approx_{H}$ is Borel reducible to $\equiv_{E_{0}}$, let $\Gamma=H \times \mathbb{Z}$ and let $\rho: \Gamma \rightarrow H$ be the canonical surjective homomorphism. Let $\nu$ be the usual product probability measure on $X=2^{\Gamma}$ and let $E=E_{\Gamma}^{2^{\Gamma}}$. Then, arguing as in the proof of Theorem 5.8, it follows that the Borel map $\pi \mapsto \sigma_{\pi}$ from $\operatorname{Rep}(H, \mathcal{H})$ to $Z^{1}\left(\Gamma \times 2^{\Gamma}, U(\mathcal{H})\right)$ given by Proposition 5.4 induces a Borel reduction from $\approx_{H}$ to $\equiv_{E}$. Recall that $E_{0}$ is the orbit equivalence relation arising from the natural free ergodic action of the direct sum $D$ of countably many copies of the cyclic 
group of order 2 on the Cantor space $2^{\mathbb{N}}$. By Ornstein-Weiss [41], since $D$ and $\Gamma$ are both amenable, the essentially free ergodic actions of $\Gamma$ on $2^{\Gamma}$ and $D$ on $2^{\mathbb{N}}$ are orbit equivalent. Applying Lemma 5.7, it follows that $\equiv_{E}$ and $\equiv_{E_{0}}$ are Borel bireducible, and hence $\approx_{H}$ is Borel reducible to $\equiv_{E_{0}}$.

Next let $G=\bigoplus_{n \in \mathbb{N}} G_{n}$, where each $G_{n}$ is isomorphic to $\operatorname{Sym}(3)$. Applying Theorem 4.1, since $G$ is locally finite, it follows that $\approx_{G}$ is Borel reducible to $\approx_{H}$. Hence, in order to show that $\equiv_{E_{0}}$ is Borel reducible to $\approx_{H}$, it is enough to show that $\equiv_{E_{0}}$ is Borel reducible to $\approx_{G}$. Express $G=A \rtimes H$, where $A=\oplus_{n \in \mathbb{N}} A_{n}$ is the direct sum of countably many copies of the cyclic group of order 3 and $H=\oplus_{n \in \mathbb{N}} H_{n}$ is the direct sum of countably many copies of the cyclic group of order 2. Then the unitary dual $Z=\operatorname{Irr}_{1}(A)$ of $A$ is the direct product of countably many copies of the cyclic group $C_{3}=\left\{1, \xi, \xi^{2}\right\}$ of order 3 . Let

$$
X=\left\{\xi, \xi^{2}\right\}^{\mathbb{N}} \subseteq C_{3}^{\mathbb{N}}=Z
$$

and let $\mu$ be the product probability measure on $X$. Then the conjugation action of $H$ on $A$ induces a free ergodic action of $H$ on $(X, \mu)$ and the corresponding orbit equivalence relation $E=E_{H}^{X}$ is clearly orbit equivalent to $E_{0}$. Hence, by Lemma 5.7, it follows that $\equiv_{E}$ and $\equiv_{E_{0}}$ are Borel bireducible. Let $\sigma \stackrel{\pi}{\mapsto} \pi_{\sigma}$ be the Borel map from $Z^{1}(H \times X, U(\mathcal{H}))$ to $\operatorname{Rep}\left(G, L^{2}(X, \mathcal{H})\right)$ given by equations (5.2) and (5.3). Then, applying Proposition 5.3 and Schur's Lemma, $\pi$ induces a Borel reduction from $\equiv_{E}$ to $\approx_{G}$ and hence $\equiv_{E_{0}}$ is Borel reducible to $\approx_{H}$.

In the remainder of this section, we will discuss the relationship between $\equiv_{E_{0}}$ and some more commonly studied Borel equivalence relations. We will begin by considering the measure equivalence relation on a suitably restricted subspace of the Polish space $\mathrm{P}\left(2^{\mathbb{N}}\right)$ of probability measures on $2^{\mathbb{N}}$. Once again, let $D$ be the direct sum of countably many copies of the cyclic group of order 2, equipped with its natural action on the Cantor space $2^{\mathbb{N}}$. Then we can define an associated action of $D$ on $\mathrm{P}\left(2^{\mathbb{N}}\right)$ by $\mu \stackrel{g}{\mapsto} g_{*} \mu$. Recall that a probability measure $\mu \in \mathrm{P}\left(2^{\mathbb{N}}\right)$ is said to be quasi-invariant if $g_{*} \mu \sim \mu$ for all $g \in D$; and if $\mu \in \mathrm{P}\left(2^{\mathbb{N}}\right)$ is quasi-invariant, then $\mu$ is said to be ergodic if every $D$-invariant Borel subset of $2^{\mathbb{N}}$ is either null or conull. It is easily checked that the set $\mathrm{QE}\left(2^{\mathbb{N}}\right)$ of quasi-invariant ergodic probability 
measures on $2^{\mathbb{N}}$ is a Borel subset of $\mathrm{P}\left(2^{\mathbb{N}}\right)$ and hence $\mathrm{QE}\left(2^{\mathbb{N}}\right)$ is a standard Borel space.

Definition 6.4. $\sim_{q e}$ is the measure equivalence relation on $\mathrm{QE}\left(2^{\mathbb{N}}\right)$.

The following result is implicitly contained in Mackey [38].

Theorem 6.5. $\sim_{q e}$ is Borel reducible to $\equiv_{E_{0}}$.

Proof. Once again, let $G=\bigoplus_{n \in \mathbb{N}} G_{n}$, where each $G_{n}$ is isomorphic to $\operatorname{Sym}(3)$; and express $G=A \rtimes H$, where $A=\oplus_{n \in \mathbb{N}} A_{n}$ is the direct sum of countably many copies of the cyclic group of order 3 and $H=\oplus_{n \in \mathbb{N}} H_{n}$ is the direct sum of countably many copies of the cyclic group of order 2 . Then it is enough to show that $\sim_{q e}$ is Borel reducible to $\approx_{G}$. As above, let $Z=\operatorname{Irr}_{1}(A)$ be the unitary dual of $A$ and let

$$
X=\left\{\xi, \xi^{2}\right\}^{\mathbb{N}} \subseteq C_{3}^{\mathbb{N}}=Z
$$

Then the induced action of $H$ on $X$ is isomorphic to the natural action of $D$ on $2^{\mathbb{N}}$. If the probability measure $\mu$ on $X$ is quasi-invariant and ergodic with respect to the action of $H$, then we can define a corresponding irreducible unitary representation $\pi_{\mu}$ of $G$ on $L^{2}(X, \mu)$ by setting:

$$
\left(\pi_{\mu}(g) \cdot f\right)(x)= \begin{cases}\sqrt{\frac{d\left(g_{*} \mu\right)}{d \mu}(x)} f\left(g^{-1} x\right) & \text { if } g \in H ; \\ x(g) f(x) & \text { if } g \in A ;\end{cases}
$$

for each $f \in L^{2}(X, \mu)$. Suppose that $\nu$ is another probability measure on $X$ which is quasi-invariant and ergodic with respect to the action of $H$. If $\mu \sim \nu$, then the unitary isomorphism $U: L^{2}(X, \mu) \rightarrow L^{2}(X, \nu)$, defined by $f \mapsto \sqrt{d \mu / d \nu} f$, satisfies

$$
U^{-1} \pi_{\nu}(g) U=\pi_{\mu}(g)
$$

for each $g \in G$ and hence $\pi_{\nu}, \pi_{\mu}$ are unitarily equivalent. Conversely, if $\pi_{\nu}, \pi_{\mu}$ are unitarily equivalent, then $\pi_{\nu} \uparrow A$ and $\pi_{\mu} \uparrow A$ are unitarily equivalent representations of $A$, and it is well-known that this implies that $\mu \sim \nu$. Thus $\sim_{q e}$ is Borel reducible to $\approx_{G}$.

Next, following Kechris-Sofronidis [34], we will discuss the relationship between the Borel equivalence relations $\sim_{q e}$ and $E_{\ell^{2}}$. 
Definition 6.6. $E_{\ell^{2}}$ is the Borel equivalence relation on $\mathbb{R}^{\mathbb{N}}$ defined by

$$
\left(a_{n}\right) E_{\ell^{2}}\left(b_{n}\right) \Longleftrightarrow \sum\left(a_{n}-b_{n}\right)^{2}<\infty
$$

In other words, $E_{\ell^{2}}$ is the orbit equivalence relation arising from the additive action of the Banach space $\ell^{2}=\left\{\left(x_{n}\right) \in \mathbb{R}^{\mathbb{N}} \mid \sum x_{n}^{2}<\infty\right\}$ on $\mathbb{R}^{\mathbb{N}}$. It is well-known that $E_{\ell^{2}}$ is Borel bireducible with $E_{\ell^{2}} \uparrow(0,1)^{\mathbb{N}}$. (For example, see the proof of Kanovei [30, Lemma 6.2.2].) As pointed out by Kechris-Sofronidis [34], the classical theorem of Kakutani [29] on equivalence of infinite product measures implies that $E_{\ell^{2}}$ is Borel reducible to $\sim_{q e}$. In more detail, for each sequence $\alpha=\left(\alpha_{n}\right) \in(0,1)^{\mathbb{N}}$, let $\mu_{\alpha}$ be the product measure defined on $2^{\mathbb{N}}$ by

$$
\mu_{\alpha}=\prod\left(\alpha_{n} \delta_{0}+\left(1-\alpha_{n}\right) \delta_{1}\right)
$$

where $\delta_{i}$ denotes the Dirac measure on $\{0,1\}$. Then it is well-known that each $\mu_{\alpha}$ is quasi-invariant and ergodic; and, by Kakutani [29], if $\alpha, \beta \in(0,1)^{\mathbb{N}}$, then the following are equivalent:

(i) $\mu_{\alpha} \sim \mu_{\beta}$.

(ii) $\sum_{n=0}^{\infty}\left(\sqrt{\alpha_{n}}-\sqrt{\beta_{n}}\right)^{2}+\left(\sqrt{1-\alpha_{n}}-\sqrt{1-\beta_{n}}\right)^{2}<\infty$.

Furthermore, if there exists $\varepsilon>0$ such that $\varepsilon \leq \alpha_{n}, \beta_{n} \leq 1-\varepsilon$ for all $n \in \mathbb{N}$, then condition (ii) is equivalent to:

(iii) $\sum_{n=0}^{\infty}\left(\alpha_{n}-\beta_{n}\right)^{2}<\infty$.

It follows easily that $E_{\ell^{2}}$ is Borel bireducible with the restriction of $\sim_{q e}$ to the space $\left\{\mu_{\alpha} \mid \alpha \in(0,1)^{\mathbb{N}}\right\}$ of quasi-invariant ergodic product measures. In particular, it is now clear that the following result holds.

Corollary 6.7. $E_{\ell^{2}}$ is Borel reducible to $\equiv_{E_{0}}$.

Remark 6.8. Suppose that $G$ is a countable non-type I group. Then the results in this section imply that $E_{\ell^{2}}$ is Borel reducible to $\approx_{G}$. By Kechris-Sofronidis [34], $E_{\ell^{2}}$ does not admit classification by countable structures; and hence we obtain yet another proof of Hjorth's theorem that $\approx_{G}$ does not admit classification by countable structures. (See also Farah [14] and Kerr-Li-Pichot [35].)

Question 6.9. Is $\sim_{q e}$ Borel bireducible with $E_{\ell^{2}}$ ? 
Question 6.10. Is $\equiv_{E_{0}}$ Borel bireducible with $E_{\ell^{2}}$ ?

While there is currently no reason to expect a positive answer to Question 6.10, a positive answer would enable us to separate $\equiv_{E_{0}}$ and $\equiv_{E_{\infty}}$. In more detail, the following result will be proved in Section 7 .

Theorem 6.11. $E_{\infty}$ is Borel reducible to $\equiv_{E_{\infty}}$.

On the other hand, as was pointed out to me by Alexander Kechris, the following result is a straightforward consequence of Popa's Superrigidity Theorem [42].

Theorem 6.12. $E_{\infty}$ is not Borel reducible to $E_{\ell^{2}}$.

Proof. Suppose that $E_{\infty}$ is Borel reducible to $E_{\ell^{2}}$. Let $\Gamma$ be a countably infinite perfect Kazhdan group (e.g. we could take $\Gamma=S L(3, \mathbb{Z})$ ) and consider the action of $\Gamma$ on $\left(2^{\Gamma}, \mu\right)$, where $\mu$ is the usual product probability measure. Then the corresponding orbit equivalence relation $E=E_{\Gamma}^{2^{\Gamma}}$ is a countable Borel equivalence relation and so $E$ is Borel reducible to $E_{\infty}$. Hence there exists a Borel reduction $f: 2^{\Gamma} \rightarrow \mathbb{R}^{\mathbb{N}}$ from $E$ to $E_{\ell^{2}}$. Let $\alpha: \Gamma \times 2^{\Gamma} \rightarrow \ell^{2}$ be the corresponding Borel cocycle defined by

$$
\alpha(\gamma, x)=\text { the unique } \bar{a} \in \ell^{2} \text { such that } f(\gamma \cdot x)=f(x)+\bar{a} .
$$

By Ando-Matsuzawa [1, Example 2.18], $\ell^{2}$ is a Polish group of finite type. Hence, applying Popa [42], it follows that $\alpha$ is equivalent to a group homomorphism; i.e. there exists a Borel map $b: 2^{\Gamma} \rightarrow \ell^{2}$ and a group homomorphism $\varphi: \Gamma \rightarrow \ell^{2}$ such that for all $\gamma \in \Gamma$,

$$
\begin{aligned}
\varphi(\gamma) & =b(\gamma \cdot x)+\alpha(\gamma, x)-b(x) \\
& =b(\gamma \cdot x)+f(\gamma \cdot x)-(b(x)+f(x)) \quad \mu \text {-a.e. } x \in 2^{\Gamma} .
\end{aligned}
$$

Since $\Gamma$ is perfect, it follows that $\varphi$ is the trivial homomorphism which sends every element of $\Gamma$ to the identity element $\overline{0} \in \ell^{2}$; and so for all $\gamma \in \Gamma$,

$$
b(\gamma \cdot x)+f(\gamma \cdot x)=b(x)+f(x) \quad \mu \text {-a.e. } x \in 2^{\Gamma} .
$$

Clearly the Borel map $f^{\prime}: 2^{\Gamma} \rightarrow \mathbb{R}^{\mathbb{N}}$ defined by $f^{\prime}(x)=b(x)+f(x)$ is also a Borel reduction from $E$ to $E_{\ell^{2}}$. However, we have just seen that $f^{\prime}$ is $\Gamma$-invariant $\mu$-a.e.; and since $\Gamma$ acts ergodically on $\left(2^{\Gamma}, \mu\right)$, this implies that $f^{\prime}$ is constant $\mu$-a.e., which is a contradiction. 


\section{QUASI-REgular REPRESENTATIONS OF $\mathbb{F}_{2}$}

In this section, we will prove that the universal countable Borel equivalence relation $E_{\infty}$ is Borel reducible to the unitary equivalence relation $\approx_{\mathbb{F}_{2}}$ on the space of infinite dimensional irreducible unitary representations of the free group $\mathbb{F}_{2}$ on the two generators $\{a, b\}$. Of course, combined with Theorem 5.8, this implies that $E_{\infty}$ is Borel reducible to $\equiv_{E_{\infty}}$. We will begin by recalling Mackey's theorem on quasi-regular representations of countable groups.

Let $G$ be a countable group and let $H \leqslant G$ be a subgroup. Then $\lambda_{G / H}$ denotes the corresponding quasi-regular representation of $G$ on the Hilbert space $\ell^{2}(G / H)$. Recall that two subgroups $H_{0}, H_{1}$ of a group $G$ are said to be commensurable if $H_{0} \cap H_{1}$ is of finite index in both $H_{0}$ and $H_{1}$. The commensurator of $H_{0}$ in $G$ is defined to be

$$
\operatorname{Com}_{G}\left(H_{0}\right)=\left\{g \in G \mid H_{0} \text { and } g H_{0} g^{-1} \text { are commensurable }\right\} \text {. }
$$

The subgroup $H_{0}$ is said to be self-commensurating if $\operatorname{Com}_{G}\left(H_{0}\right)=H_{0}$. It is easily seen that if $H_{0}$ and $H_{1}$ are self-commensurating subgroups of $G$, then $H_{0}$ and $H_{1}$ are conjugate in $G$ if and only if there exists an element $g \in G$ such that $g H_{0} g^{-1}$ and $H_{1}$ are commensurable. Thus the following result is an immediate consequence of Corollary 1 and Corollary 2 in Mackey [38, Section 3.4]. (The formulation of Theorem 7.1 is a slight variant of Theorem 2.1 in Burger and de la Harpe [7].)

Theorem 7.1 (Mackey). Let $G$ be a countable group and let $H_{0}, H_{1}$ be subgroups of $G$.

(a) $\lambda_{G / H_{0}}$ is irreducible if and only if $H_{0}$ is a self-commensurating subgroup.

(b) If $H_{0}$ and $H_{1}$ are self-commensurating subgroups, then $\lambda_{G / H_{0}}$ is unitarily equivalent to $\lambda_{G / H_{1}}$ if and only if $H_{0}$ and $H_{1}$ are conjugate in $G$.

Remark 7.2. Suppose that $G$ is a countable group and that $H \leqslant G$ is a selfcommensurating subgroup. Extending the results of Mackey [38, Section 3.4] on inducing 1-dimensional representations of $H$, Corwin [8] and Kleppner [36] proved that if $\pi, \sigma$ are finite dimensional irreducible representations of $H$, then:

(i) $\operatorname{ind}_{H}^{G}(\pi)$ is an irreducible representation of $G$; and

(ii) $\pi \approx_{H} \sigma$ if and only if $\operatorname{ind}_{H}^{G}(\pi) \approx_{G} \operatorname{ind}_{H}^{G}(\sigma)$. 
For many years, it was unknown whether or not the analogous statements were also true for infinite dimensional irreducible representations $\pi, \sigma \in \operatorname{Irr}_{\infty}(H)$. However, Bekka-Curtis [4] have recently shown that there exists a self-commensurating subgroup $H \leqslant G=\mathbb{Z}^{2} \rtimes S L(2, \mathbb{Z})$ and an infinite dimensional irreducible representation $\pi \in \operatorname{Irr}_{\infty}(H)$ such that the induced representation $\operatorname{ind}_{H}^{G}(\pi)$ is not irreducible. It would be very interesting to find sufficient conditions on pairs $H \leqslant G$ of countable groups which ensure that the map $\pi \mapsto \operatorname{ind}_{H}^{G}(\pi)$ is a Borel reduction from $\approx_{H}$ to $\approx_{G}$.

Definition 7.3. If $G$ is a countable group, then

$$
\mathcal{C}(G)=\{H \leq G \mid H \text { is a self-commensurating subgroup }\}
$$

and $E_{\mathcal{C}(G)}$ denotes the conjugacy relation on $\mathcal{C}(G)$.

The following result is an immediate consequence of Theorem 7.1.

Lemma 7.4. $E_{\mathcal{C}\left(\mathbb{F}_{2}\right)}$ is Borel reducible to $\approx_{\mathbb{F}_{2}}$.

Thus, in order to show that $E_{\infty}$ is Borel reducible to $\approx_{\mathbb{F}_{2}}$, it is enough to prove the following result.

Theorem 7.5. $E_{\mathcal{C}\left(\mathbb{F}_{2}\right)}$ is a universal countable Borel equivalence relation.

Let $\mathbb{F}_{3}$ be the free group on the three generators $\{a, b, c\}$. Then the following easy observation shows that it is enough to prove that $E_{\mathcal{C}\left(\mathbb{F}_{3}\right)}$ is a universal countable Borel equivalence relation.

Lemma 7.6. $E_{\mathcal{C}\left(\mathbb{F}_{3}\right)}$ is Borel reducible to $E_{\mathcal{C}\left(\mathbb{F}_{2}\right)}$.

Proof. By Karrass-Solitar [31, p. 950], the set $\left\{a b a, a^{2} b^{2} a^{2}, a^{3} b^{3} a^{3}\right\}$ freely generates a malnormal subgroup $K$ of $\mathbb{F}_{2}$. (Recall that a subgroup $K$ of a group $G$ is said to be malnormal if $g K^{-1} \cap K=1$ for all $g \in G \backslash K$.) In particular, it follows that each $H \in \mathcal{C}(K)$ is also self-commensurating in $\mathbb{F}_{2}$. Furthermore, if $H_{0}, H_{1} \in \mathcal{C}(K)$ and $g \in \mathbb{F}_{2}$ satisfies $g H_{0} g^{-1}=H_{1}$, then $g \in K$. Thus the identity map is a Borel reduction from $E_{\mathcal{C}(K)}$ to $E_{\mathcal{C}\left(\mathbb{F}_{2}\right)}$.

From now on, let $\mathcal{P}_{\infty}\left(\mathbb{F}_{2}\right)$ be the standard Borel space of infinite subsets of $\mathbb{F}_{2}$. Then it is easily seen that $\left(E_{\infty}\left\lceil\mathcal{P}_{\infty}\left(\mathbb{F}_{2}\right)\right) \sim_{B} E_{\infty}\right.$ and thus $E_{\infty}\left\lceil\mathcal{P}_{\infty}\left(\mathbb{F}_{2}\right)\right.$ is also 
a universal countable Borel equivalence relation. For each $A \in \mathcal{P}_{\infty}\left(\mathbb{F}_{2}\right)$, let $K(A)$ and $S(A)$ be the subgroups of $\mathbb{F}_{3}$ defined by:

- $K(A)=\left\langle w c w^{-1} \mid w \in A\right\rangle ;$ and

- $S(A)=\left\{g \in \mathbb{F}_{2} \mid g A=A\right\}$.

Let $C=\langle c\rangle$. Then it is easily checked that

$$
K(A)=\underset{w \in A}{*} w C w^{-1}
$$

is the free product of the subgroups $\left\{w C w^{-1} \mid w \in A\right\}$. In particular, it follows that $K(A) \cap \mathbb{F}_{2}=1$. It is also clear that if $g \in S(A)$, then $g K(A) g^{-1}=K(A)$. Hence the subgroup $H(A) \leqslant \mathbb{F}_{3}$ generated by $K(A) \cup S(A)$ decomposes into a semi-direct product:

- $H(A)=K(A) \rtimes S(A)$.

Proposition 7.7. The map $A \mapsto H(A)$ is a Borel reduction from $E_{\infty}\left\lceil\mathcal{P}_{\infty}\left(\mathbb{F}_{2}\right)\right.$ to $E_{\mathcal{C}\left(\mathbb{F}_{3}\right)} \cdot$

The proof of Proposition 7.7 will be broken down into a sequence of lemmas. We will begin with the following easy observation.

Lemma 7.8. If $A \in \mathcal{P}_{\infty}\left(\mathbb{F}_{2}\right)$ and $g \in \mathbb{F}_{2}$, then $g H(A) g^{-1}=H(g A)$.

Proof. Clearly $g K(A) g^{-1}=K(g A)$ and $g S(A) g^{-1}=S(g A)$.

Most of our efforts will go into proving the following somewhat technical result.

Lemma 7.9. Suppose that $A, B \in \mathcal{P}_{\infty}\left(\mathbb{F}_{2}\right)$ and that $\gamma \in \mathbb{F}_{3}$. If $H(A)$ and $\gamma H(B) \gamma^{-1}$ are commensurable, then there exist elements $g \in \mathbb{F}_{2}$ and $u \in K(B)$ such that $\gamma=g u$.

Before presenting the proof of Lemma 7.9, we will show how to complete the proof of Proposition 7.7.

Lemma 7.10. If $A \in \mathcal{P}_{\infty}\left(\mathbb{F}_{2}\right)$, then $H(A) \in \mathcal{C}\left(\mathbb{F}_{3}\right)$.

Proof. Suppose that $\gamma \in \operatorname{Com}_{\mathbb{F}_{3}}(H(A))$. Then, by Lemma 7.9, there exist $g \in \mathbb{F}_{2}$ and $u \in K(A)$ such that $\gamma=g u$. Thus it is enough to show that $g \in S(A)$. To see this, first note that

$$
\gamma H(A) \gamma^{-1}=g H(A) g^{-1}=H(g A)
$$


Thus $H(A)$ and $H(g A)$ are commensurable; and this easily implies that $H(A)$ and $H\left(g^{-1} A\right)$ are also commensurable. Now suppose that $g A \neq A$. Then, after replacing $g$ by $g^{-1}$ if necessary, we can suppose that there exists $w_{0} \in g A \backslash A$. Since $H(A)$ and $H(g A)$ are commensurable, there exists $n \geq 1$ such that

$$
w_{0} c^{n} w_{0}^{-1} \in H(A)=K(A) \rtimes S(A) .
$$

Thus there exist $w_{1}, \cdots, w_{\ell} \in A, m_{1}, \cdots, m_{\ell} \in \mathbb{Z} \backslash\{0\}$ and $h \in S(A)$ such that

$$
w_{0} c^{n} w_{0}^{-1}=w_{1} c^{m_{1}} w_{1}^{-1} \cdots w_{\ell} c^{m_{\ell}} w_{\ell}^{-1} h .
$$

By considering the homomorphism $\mathbb{F}_{3} \rightarrow \mathbb{F}_{2}$ such that $a \mapsto a, b \mapsto b$ and $c \mapsto 1$, we see that $h=1$. But then equation (7.10) contradicts the fact that $K\left(\mathbb{F}_{2}\right)$ is the free product of the subgroups $\left\{w C w^{-1} \mid w \in \mathbb{F}_{2}\right\}$.

Lemma 7.11. Suppose that $A, B \in \mathcal{P}_{\infty}\left(\mathbb{F}_{2}\right)$. If $H(A)$ and $H(B)$ are conjugate in $\mathbb{F}_{3}$, then there exists $g \in \mathbb{F}_{2}$ such that $A=g B$.

Proof. Suppose that $\gamma \in \mathbb{F}_{3}$ and that $H(A)=\gamma H(B) \gamma^{-1}$. Then, by Lemma 7.9, there exist $g \in \mathbb{F}_{2}$ and $u \in K(B)$ such that $\gamma=g u$. Thus $H(A)=H(g B)$; and arguing as in the proof of Lemma 7.10, we see that $A=g B$.

Proof of Proposition 7.7. By Lemma 7.10, if $A \in \mathcal{P}_{\infty}\left(\mathbb{F}_{2}\right)$, then $H(A) \in \mathcal{C}\left(\mathbb{F}_{3}\right)$. Suppose that $A, B \in \mathcal{P}_{\infty}\left(\mathbb{F}_{2}\right)$. By Lemma 7.8, if there exists $g \in \mathbb{F}_{2}$ such that $g A=B$, then $g H(A) g^{-1}=H(B)$. Conversely, by Lemma 7.11, if $H(A)$ and $H(B)$ are conjugate in $\mathbb{F}_{3}$, then there exists $g \in \mathbb{F}_{2}$ such that $A=g B$. Thus the map $A \mapsto H(A)$ is a Borel reduction from $E_{\infty} \uparrow \mathcal{P}_{\infty}\left(\mathbb{F}_{2}\right)$ to $E_{\mathcal{C}\left(\mathbb{F}_{3}\right)}$.

The following argument is closely based upon the proof of Gao [19, Lemma 2].

Proof of Lemma 7.9. Suppose that $A, B \in \mathcal{P}_{\infty}\left(\mathbb{F}_{2}\right)$ and that $\gamma \in \mathbb{F}_{3}$ is such that $H(A)$ and $\gamma H(B) \gamma^{-1}$ are commensurable. Since

$$
\left[K(A): K(A) \cap \gamma H(B) \gamma^{-1}\right]<\infty
$$

it follows that for each $x \in A$, there exists $n_{x} \geq 1$ such that

$$
x c^{n_{x}} x^{-1}=\gamma w_{x} g \gamma^{-1}
$$


for some $w_{x} \in K(B)$ and $g \in S(B)$. Notice that $x c^{n_{x}} x^{-1}$ is a freely reduced word in $a, b, c$. In considering the right-hand side of equation (7.9), we will initially suppose that:

(i) $\gamma, \gamma^{-1}$ and $g$ are freely reduced words in $a, b, c$; and

(ii) $w_{x} \in K(B)$ is written as a reduced word with respect to the decomposition of $K(B)$ as the free product of the subgroups $\left\{b c b^{-1} \mid b \in B\right\}$; say,

$$
w_{x}=y_{1} \cdots y_{t}
$$

where each $y_{i}=b_{i} c^{\ell_{i}} b_{i}^{-1}$ for some $b_{i} \in B$ and $\ell_{i} \in \mathbb{Z} \backslash\{0\}$.

Then after freely reducing the initial expression of $\gamma w_{x} g \gamma^{-1}$ through successive cancellations of terms of the form $z z^{-1}$ or $z^{-1} z$ for some $z \in\{a, b, c\}$, we must eventually obtain the reduced word $x c^{n_{x}} x^{-1}$. From now on, for each $x \in A$, we will fix such a cancellation procedure. Note that for each $x \in A$, there exists an occurrence of $c$ in the initial expression of $\gamma w_{x} g \gamma^{-1}$ which is preserved throughout the cancellation procedure and gives rise to the first occurrence of $c$ in the reduced word $x c^{n_{x}} x^{-1}$. This occurrence of $c$ in $\gamma w_{x} g \gamma^{-1}$ will be called the first preserved occurrence.

We claim that there exists at most one $x \in A$ such that the corresponding first preserved occurrence of $c$ is a letter in $\gamma$. To see this, suppose that $x_{1}, x_{2} \in A$ both have this property. Since $\gamma$ is freely reduced, the first preserved occurrence must be the first $c$ in $\gamma$. Thus writing $\gamma=k c u$, where $k \in \mathbb{F}_{2}$ and $u \in \mathbb{F}_{3}$, we have that

$$
\begin{aligned}
& x_{1} c^{n_{x_{1}}} x_{1}^{-1}=k c u w_{x_{1}} g \gamma^{-1} \\
& x_{2} c^{n_{x_{2}}} x_{2}^{-1}=k c u w_{x_{2}} g \gamma^{-1},
\end{aligned}
$$

which implies that $x_{1}=k=x_{2}$. Similarly, we claim there exists at most one $x \in A$ such that the corresponding first preserved occurrence of $c$ is a letter in $\gamma^{-1}$. To see this, note if the first preserved occurrence of $c$ is a letter in $\gamma^{-1}$, then the last preserved occurrence of $c$ must also be a letter in $\gamma^{-1}$; and since $\gamma^{-1}$ is freely reduced, this last preserved occurrence must be the last $c$ in $\gamma^{-1}$. Arguing as above, the claim now follows easily.

Thus there exists $x \in A$ such that the corresponding first preserved occurrence of $c$ is a letter in $w_{x} g$. Of course, since $g \in S(B) \leqslant \mathbb{F}_{2}$, this means that the 
corresponding first preserved occurrence of $c$ is actually a letter in $w_{x}$; say, the first preserved occurrence is one of the letters in $y_{i}=b_{i} c^{\ell_{i}} b_{i}^{-1}$. Then

$$
x=\gamma b_{1} c^{\ell_{1}} b_{1}^{-1} \cdots b_{i-1} c^{\ell_{i-1}} b_{i-1}^{-1} b_{i} c^{r}
$$

for some $r \geq 0$. Let $u=b_{1} c^{\ell_{1}} b_{1}^{-1} \cdots b_{i-1} c^{\ell_{i-1}} b_{i-1}^{-1}$. Then $u \in K(B)$. If $r=0$, then $\gamma=x b_{i}^{-1} u^{-1}$ has the required form, since $x b_{i}^{-1} \in \mathbb{F}_{2}$ and $u^{-1} \in K(B)$. So suppose that $r>0$. Then we can write $x=\gamma z b_{i}$, where $z=u b_{i} c^{r} b_{i}^{-1} \in K(B)$; and, once again, $\gamma$ has the required form.

\section{UNITARY EQUIVALENCE OF ARBITRARY REPRESENTATIONS}

In the previous sections of this paper, we have focused our attention on the unitary equivalence relation $\approx_{G}$ on the space of irreducible unitary representations of the countable group $G$ on the infinite dimensional separable Hilbert space $\mathcal{H}$. In this final section, we will point out a basic open question concerning the unitary equivalence relation $\approx_{G}^{+}$on the $\operatorname{space} \operatorname{Rep}(G, \mathcal{H})$ of arbitrary unitary representations of $G$ on $\mathcal{H}$. By a recent result of Hjorth-Törnquist [26], $\approx_{G}^{+}$is a Borel equivalence relation; in fact, $\approx_{G}^{+}$is an $F_{\sigma \delta}$ equivalence relation.

Let $\sim$ be the measure equivalence relation on the space $\mathrm{P}\left(2^{\mathbb{N}}\right)$ of arbitrary probability measures on $2^{\mathbb{N}}$. (Of course, we could replace $2^{\mathbb{N}}$ with any uncountable standard Borel space $X$.) Then, as pointed out by Kechris [33, Section 5], the spectral theorem for unitary operators implies that the unitary equivalence relation $\approx_{\mathbb{Z}}^{+}$ on the space $\operatorname{Rep}(\mathbb{Z}, \mathcal{H})$ is Borel bireducible with $\sim$. In particular, it follows that $\approx_{\mathbb{Z}}^{+}$is not smooth and hence $\approx_{\mathbb{Z}}<_{B} \approx_{\mathbb{Z}}^{+}$. More generally, the following result is a straightforward consequence of Kanovei's theory [30, Chapter 17] of pinned Borel equivalence relations.

Theorem 8.1 (Hjorth-Törnquist [26]). If $G$ is a countably infinite group, then $\approx_{G}<_{B} \approx_{G}^{+}$

However, the following basic problem remains open.

Question 8.2. Is $\approx_{\mathbb{F}_{\infty}}^{+}$Borel bireducible with $\approx_{\mathbb{Z}}^{+}$? Equivalently, is $\approx_{\mathbb{F}_{\infty}}^{+}$Borel bireducible with the measure equivalence relation $\sim$ ? 
Here it is probably worth pointing out that $E_{\infty}$ is Borel reducible to $\sim$ and hence $E_{\infty}$ is Borel reducible to $\approx_{\mathbb{Z}}^{+}$. In fact, I am currently not aware of any even vaguely plausible strategy for separating $\approx_{\mathbb{Z}}^{+}$and $\approx_{\mathbb{F}_{\infty}}^{+}$.

\section{Appendix A. List of EQUivalence Relations}

In this appendix, we collect together the definitions of the various Borel equivalence relations that occur in this paper.

- $\approx_{G}$ is the unitary equivalence relation on the space $\operatorname{Irr}_{\infty}(G)$ of infinite dimensional irreducible unitary representations of the countable non-type I group $G$.

- $\approx_{G}^{+}$is the unitary equivalence relation on the $\operatorname{space} \operatorname{Rep}(G, \mathcal{H})$ of arbitrary unitary representations of the countable group $G$ on the infinite dimensional separable Hilbert space $\mathcal{H}$.

- $\approx_{\mathcal{A}}$ is the unitary equivalence relation on the $\operatorname{space} \operatorname{Irr}_{\infty}(\mathcal{A})$ of infinite dimensional irreducible unitary representations of the non-type I separable $C^{*}$-algebra $\mathcal{A}$.

- $E_{G}^{X}$ is the orbit equivalence relation of the countable group $G$ acting on the standard Borel $G$-space $X$.

- $E_{0}$ is the eventual equality relation on $2^{\mathbb{N}}$.

- $E_{\infty}$ is the orbit equivalence relation of the shift action of $\mathbb{F}_{2}$ on $2^{\mathbb{F}_{2}}$.

- $\equiv_{E}$ is the orbit equivalence relation of the action of $L(X, U(\mathcal{H}))$ on the space $\operatorname{Irr}(E, U(\mathcal{H}))$ of irreducible cocycles.

- $\sim$ is the measure equivalence relation on the space $\mathrm{P}(X)$ of probability measures on the uncountable Polish space $X$.

- $\sim_{q e}$ is the measure equivalence relation on the space $\mathrm{QE}\left(2^{\mathbb{N}}\right)$ of quasiinvariant ergodic probability measures on $2^{\mathbb{N}}$.

- $E_{\ell^{2}}$ is the orbit equivalence relation arising from the additive action of $\ell^{2}=\left\{\left(x_{n}\right) \in \mathbb{R}^{\mathbb{N}} \mid \sum x_{n}^{2}<\infty\right\}$ on $\mathbb{R}^{\mathbb{N}}$.

- $E_{\mathcal{C}(G)}$ is the conjugacy relation on the space $\mathcal{C}(G)$ of self-commensurating subgroups of the countable group $G$. 


\section{REFERENCES}

[1] H. Ando and Y. Matsuzawa, On Polish Groups of Finite Type, Publ. Res. Inst. Math. Sci. 48 (2012), 389-408.

[2] W. Arveson, An invitation to $C^{*}$-algebras, Graduate Texts in Mathematics 39, SpringerVerlag, New York, 1976.

[3] R. Baer, Abelian groups without elements of finite order, Duke Math. Journal 3 (1937), $68-122$.

[4] M. Bekka and R. Curtis, On Mackey's irreducibility criterion for induced representations, Int. Math. Res. Not. 38 (2003), 2095-2101.

[5] L. Bowen, Stable orbit equivalence of Bernoulli shifts over free groups, Groups Geom. Dyn. 5 (2011), 17-38.

[6] N.P. Brown and N. Ozawa, $C^{*}$-Algebras and Finite-Dimensional Approximations, Graduate Studies in Mathematics 88, American Mathematical Society, Providence, RI, 2008.

[7] M. Burger and P. de la Harpe, Constructing irreducible representations of discrete groups, Proc. Ind. Acad. Sci. (Math. Sci.) 107 (1997), 223-235.

[8] L. Corwin, Induced representations of discrete groups, Proc. Amer. Math. Soc. 47 (1975), $279-287$.

[9] J. Dixmier, $C^{*}$-algebras, North-Holland, Amsterdam, 1977.

[10] R. Dougherty, S. Jackson and A. S. Kechris, The structure of hyperfinite Borel equivalence relations, Trans. Amer. Math. Soc. 341 (1994), 193-225.

[11] G. A. Elliott, On the classification of inductive limits of sequences of semisimple finitedimensional algebras, J. Algebra 38 (1976), 29-44.

[12] G. A. Elliott, The Mackey Borel structure on the spectrum of an approximately finitedimensional separable $C^{*}$-algebra, Trans. Amer. Math. Soc. 233 (1977), 59-68.

[13] I. Epstein, Orbit inequivalent actions of non-amenable groups, preprint (2007).

[14] I. Farah, A Dichotomy for the Makey Borel Structure, in: Proceedings of the 11th Asian Logic Conference, World Scientific, 2011, pp. 86-93.

[15] I. Farah, A. S. Toms and A. Törnquist, The descriptive set theory of $C^{*}$-algebra invariants, to appear in Int. Math. Res. Not. IMRN.

[16] J. Feldman and C. C. Moore, Ergodic equivalence relations, cohomology and von Neumann algebras I, Trans. Amer. Math. Soc. 234 (1977), 289-324.

[17] G. B. Folland, A Course in Abstract Harmonic Analysis, CRS Press, 1995

[18] D. Gaboriau and R. Lyons, A measurable-group-theoretic solution to von Neumann's problem, Invent. Math. 117 (2009), 533-540.

[19] S. Gao, Coding subset shifts by subgroup conjugacy, Bull. London Math. Soc. 32 (2000), $653-657$.

[20] S. Gao, Invariant descriptive set theory, CRC Press, Boca Raton, 2009.

[21] J. Glimm, Type I $C^{*}$-algebras, Ann. of Math. 73 (1961), 572-612. 
[22] L. Harrington, A. S. Kechris and A. Louveau, A Glimm-Effros dichotomy for Borel equivalence relations, J. Amer. Math. Soc. 3 (1990), 903-927.

[23] E. Hewitt and K. Stromberg, Real and abstract analysis, Springer-Verlag, New York, 1965.

[24] G. Hjorth, Non-smooth infinite dimensional group representations, preprint (1997).

[25] G. Hjorth, Classification and Orbit Equivalence Relations, Mathematical Surveys and Monographs, vol. 75, American Mathematical Society, Providence, RI, 2000.

[26] G. Hjorth and A. Törnquist, The conjugacy relation on unitary representations, Math. Res. Lett. 19 (2012), no. 3, 525535.

[27] B. Huppert, Character theory of finite groups, de Gruyter Expositions in Mathematics 25, Walter de Gruyter, Berlin, 1998.

[28] S. Jackson, A.S. Kechris, and A. Louveau, Countable Borel equivalence relations, J. Math. Logic 2 (2002), 1-80.

[29] S. Kakutani, On equivalence of infinite product measures, Ann. of Math. 49 (1948), 214-224.

[30] V. Kanovei, Borel equivalence relations: Structure and classification, University Lecture Series, vol. 44, American Mathematical Society, Providence, 2008.

[31] A. Karrass and D. Solitar, The free product of two groups with a malnormal amalgamated subgroup Canad. J. Math. 23 (1971), 933-959.

[32] A. S. Kechris Classical Descriptive Set Theory, Graduate Texts in Mathematics 156, Springer-Verlag, 1995

[33] A. S. Kechris, Global aspects of ergodic group actions, Mathematical Surveys and Monographs, vol. 160, American Mathematical Society, Providence, RI, 2010.

[34] A. S. Kechris and N. E. Sofronidis, A strong generic ergodicity property of unitary and selfadjoint operators, Ergodic Theory Dynam. Systems 21 (2001), 1459-1479.

[35] D. Kerr, David, H. Li and M. Pichot, Turbulence, representations, and trace-preserving actions, Proc. Lond. Math. Soc. (3) 100 (2010), 459-484.

[36] A. Kleppner, On the intertwining number theorem, Proc. Amer. Math. Soc. 12 (1961), 731733.

[37] A. J. Lazar and D.C. Taylor, Approximately finite-dimensional $C^{*}$-algebras and Bratteli diagrams, Trans. Amer. Math. Soc. 259 (1980), 599-619.

[38] G.W. Mackey, The theory of unitary group representations, The University of Chicago Press, 1976.

[39] L. Motto Ros, On the complexity of the relations of isomorphism and bi-embeddability, Proc. Amer. Math. Soc. 140 (2012), 309-323.

[40] O. A. Nielsen, Direct integral theory, Lecture Notes in Pure and Applied Mathematics, vol. 61, Marcel Dekker, New York, 1980.

[41] D. S. Ornstein and B. Weiss, Ergodic theory of amenable group actions I: The Rohlin lemma, Bull. Amer. Math. Soc. 2 (1980), 161-164.

[42] S. Popa, Cocycle and orbit equivalence superrigidity for malleable actions of w-rigid groups, Invent. Math. 170 (2007), 243-295. 
[43] J. C. Quigg, On the irreducibility of an induced representation, Pacific J. Math. 93 (1981), $163-179$.

[44] J. P. Serre, Trees, Springer-Verlag, 1980.

[45] C. E. Sutherland, On a construction of unitary cocycles and the representation theory of amenable groups, Ergodic Theory Dynam. Systems 3 (1983), 129-135.

[46] E. Thoma, Über unitäre Darstellungen abzählbarer discreter Gruppen, Math. Ann. 153 (1964), 111-138.

Mathematics Department, Rutgers University, 110 Frelinghuysen Road, Piscataway, NEW JERSEy 08854-8019, USA

E-mail address: sthomas@math.rutgers.edu 